\title{
Near-Infrared Sub-Bandgap All-Silicon Photodetectors: A Review
}

\author{
M. Casalino \\ Consiglio Nazionale delle Ricerche, Istituto per la Microelettronica e Microsistemi, Napoli, 80131, Italy
}

\begin{abstract}
Silicon Photonics has generated an increasing interest in recent years both for optical telecommunications and for optical interconnects in microelectronic circuits. Although silicon photodiodes are excellent detectors at visible wavelengths, due to its band-gap the development of high-performance photodetectors at near infrared wavelengths has remained an imperative but unaccomplished task so far. In recent years, however, a number of near-infrared all-silicon photodetectors have been proposed and demonstrated useful in many application fields. In this paper, a review of the state of the art is presented. Devices based on mid-bandgap absorption, surface-state absorption, internal photoemission absorption and two-photon absorption are reported, their working principles elucidated and their performance discussed and compared.
\end{abstract}

Keywords Photodetector, Silicon, Absorption, Near-Infrared Wavelengths

\section{Introduction}

Silicon Photonics has emerged as an interesting field due to its potential for low-cost optical components integrated with electronic functionality. In the past two decades, there has been growing interest in photonic devices based on Si-compatible materials[1,2] in the field both of the optical telecommunications and of the optical interconnects. In this contest, tremendous progresses in the technological processes based on the use silicon-on-insulator (SOI) substrates have allowed to obtain reliable and effectiveness full complementary metal-oxide semiconductor (CMOS) compatible optical components such as, low loss waveguides, high-Q resonators, high speed modulators, couplers, and optically pumped lasers[3-8]. All these devices have been developed to operate in the wavelength range from $\mathrm{C}$ optical band $(1528-1561 \mathrm{~nm})$ to $\mathrm{L}$ optical band (1561-1620 nm). However one of the crucial steps toward the integration of photonics with electronics resides in the development of efficient chip-scale photodetectors (PD) integrated on Si. Bulk photodetectors are perhaps the oldest and best understood silicon optoelectronic devices. Commercial products in $\mathrm{Si}$ operate at wavelengths below $1100 \mathrm{~nm}$, where band-toband absorption occurs. For the realization of photodiodes integrated in photonics circuits operating at wavelengths beyond $1100 \mathrm{~nm}$ silicon is not the right material because its transparency. However, in recent years, in order to take advantage of low-cost standard Si-CMOS processing technology, a number of photodetectors have been proposed

* Corresponding author:

maurizio.casalino@na.imm.cnr.it (M. Casalino)

Published online at http://journal.sapub.org/optics

Copyright (C) 2012 Scientific \& Academic Publishing. All Rights Reserved based on different physical effects, such as: mid-bandgap absorption (MBA), surface-state absorption (SSA), internal photoemission absorption (IPA) and two-photon absorption (TPA). In this work an overview of the state of the art on near-infrared (NIR) sub-bandgap all-silicon photodetectors is presented. First, the physical effects and the working principles of these devices are elucidated. Then, the main structures reported in the literature and the most significant results obtained in recent years are reviewed and discussed, comparing the performance of devices based on different approaches.

\section{Photodetector Performances}

It is useful to briefly recall the main performance requirements of integrated photodetectors for NIR optical applications. High speed, high responsivity, low dark current, low bias voltage and small dimensions are appealing properties for a photodetector and research efforts are now aimed at achieving all these in single devices.

As bandwidth demand keeps increasing, it is essential that all-silicon photodetectors operate at $5 \mathrm{GHz}$, a frequency at which hybrid detectors have already been demonstrated[9]. Of course, higher bandwidths $(20 \mathrm{GHz},>50 \mathrm{GHz})$ are desirable in order to anticipate future trends in optical interconnects. In fact, very high-speed photodetectors, combined with dense wavelength division multiplexing (DWDM) technology in the $\mathrm{C}$ band $(1528-1561 \mathrm{~nm})$ and $\mathrm{L}$ band (1561-1620 nm)[10], have the potential to achieve a bandwidth greater than $0.5 \mathrm{THz}$.

Another important property of a detector is described by its responsivity, which indicates the current produced by a certain optical power. Reasonable responsivities are neces- 
sary for an acceptable signal-to-noise ratio and to ease the design and realization of the amplifier circuitry that follows. Responsivity is strictly linked to a device's quantum efficiency, a property describing how many carriers per photon are collected. It is worth noting that there is a difference between internal and external quantum efficiency: in the case of internal quantum efficiency, the number of carriers that contribute to the photocurrent is related to the number of absorbed photons, while in the case of external quantum efficiency, they are related to the number of incident photons. In the telecommunications field a responsivity $\geq 0.1 \mathrm{~A} / \mathrm{W}[11]$, corresponding to external quantum efficiencies $\eta$ of $15 \%$, $10 \%$, and $8 \%$ at $\lambda=850,1300$, and $1550 \mathrm{~nm}$, respectively, is required.

In order to evaluate detector performance, an important property is dark current. This is a serious issue because the shot noise, associated with the fluctuations in a measured signal due to the random arrival time of the particles carrying energy, generates a leakage current which can increase the bit error rate (BER). Dark current depends on work frequency and it is worth noting that a higher dark current could be allowed if a system worked at frequencies at which the amplifier noise overcame photodetector noise. In a typical photodetector, dark currents less than $1 \mu \mathrm{A}$ are required.

A further requirement of photodetectors is low-voltage operation. It would be desirable to realize devices operating at the same power supply as the CMOS circuitry, i.e., bias voltage $<5 \mathrm{~V}$ and as low as $1 \mathrm{~V}$ for advanced CMOS generation.

Finally, the shrinking of photodetector dimensions would allow for the integration of photonic components with integrated electronic circuits, enabling interconnection bandwidths that are not limited by the RC time constant or the reliability constraints of metal lines.

\section{NIR Silicon Absorption Physics}

In order to develop NIR all-silicon photodetectors while taking advantage of low-cost standard silicon processing technology without additional material or process steps, a number of options have been proposed. In this section we elucidate the physical effects allowing absorption at NIR wavelengths in silicon.

\subsection{Mid-Bandgap Absorption (MBA)}

In 1959 H. Y. Fan and A. K. Ramdas[12] reported that radiation damaged $\mathrm{Si}$ would produce a photocurrent when illuminated with sub bandgap radiation.

Silicon electrical properties have been investigated after high energy particle irradiation for electrons of various energies[13,14], 9.6 MeV deuterons[15,16], and reactor fast neutrons $[17,18]$. It was demonstrated that irradiations remove charge carriers both in $\mathrm{n}$ - and in p-types $\mathrm{Si}$, and due to the introduction of both donor or acceptor defects the semiconductor becomes nearly intrinsic under prolonged irradiation. The defects electronic energy levels can be de- termined by several measurements: the temperature variation of the Hall coefficient, the rate of carrier removal as a function of the Fermi level or carrier concentration and the carrier lifetime behavior after irradiation as a function of the temperature.

Irradiation by deuteron and electron show energy levels are introduced about at $0.03 \mathrm{eV}$ below the conduction band and at $0.05 \mathrm{eV}$ above the valence band. It has been shown that the defect centers, from which the two levels are arisen, are introduced at similar rates and of the same order of magnitude as the estimated rate of displacement of atoms whose displacement energy for silicon has been reported to be $12.8 \mathrm{eV}[19]$. Production rates of the other levels, instead, results much lower.

Interstitial atoms and vacancies seem associated to two levels $\left(\mathrm{E}_{\mathrm{C}}-0.03 \mathrm{eV}\right.$ and $\left.\mathrm{E}_{\mathrm{V}}+0.05 \mathrm{eV}\right)$ but are not the unique levels introduced, in fact, there are more levels lying deeper in the energy gap. Studies of carrier mobility in electron bombarded samples drove Wertheim[13] to propose a model in which the $\mathrm{E}_{\mathrm{C}}-0.16 \mathrm{eV}$ and $\mathrm{E}_{\mathrm{V}}+0.27 \mathrm{eV}$ levels are associated with defects consisting of an interstitial atom and a vacancy separated by $2.5 \AA$ and $50 \AA$, respectively. Two levels arise from the donor and acceptor member of the pair, respectively. Moreover studies on minority carrier lifetime in $\mathrm{n}$ - and p-type samples lead to conclude that there is a spectrum of levels extending from $\mathrm{E}_{\mathrm{C}}-0.16 \mathrm{eV}$ toward the middle of the energy gap.

Unfortunately Hill's results on the effect of electron irradiation on the carrier mobility[14] show a discrepancy from the results of above cited model, for this reason the model should be object of further investigation.

Some effects of irradiation on the silicon optical properties have been observed. The absorption increased in the region of the intrinsic absorption edge of not irradiated silicon. New absorption bands are introduced at infrared wavelength and, in particular, in the near infrared the bands have peak absorption at $1.8 \mu \mathrm{m}$. Because the free carrier absorption, as expected, has been reduced due to the irradiation, this effect may greatly enhance the infrared transmission in samples of initially high carrier concentrations and facilitates the investigation of irradiation produced absorption bands in such samples.

Curve A in Fig. 1 shows the intrinsic absorption edge in normal, high resistivity silicon. Curves $\mathrm{B}$ and $\mathrm{C}$ are two of the curves obtained on a sample after successive deuteron irradiations[12].

Due to the irradiation effect, at wavelength less than $1 \mu \mathrm{m}$, an apparent shift of the absorption edge toward lower energy can be noted. The shift could be caused by a modification of the band structure due to the presence of defects. Under prolonged irradiation the effect saturates after a shift of about $0.1 \mathrm{eV}$. The absorption in the irradiated sample extends up to 2 or $3 \mu \mathrm{m}$.

The introduction of mid-band-gap energy levels via defect incorporation into the silicon lattice in order to realize photonic devices is often realized by a $\mathrm{Si}+$ ion implantation. The mechanism for the absorption at infrared wavelength is 
always the same: the introduction of defects, thus of energy levels inside the silicon bandgap.

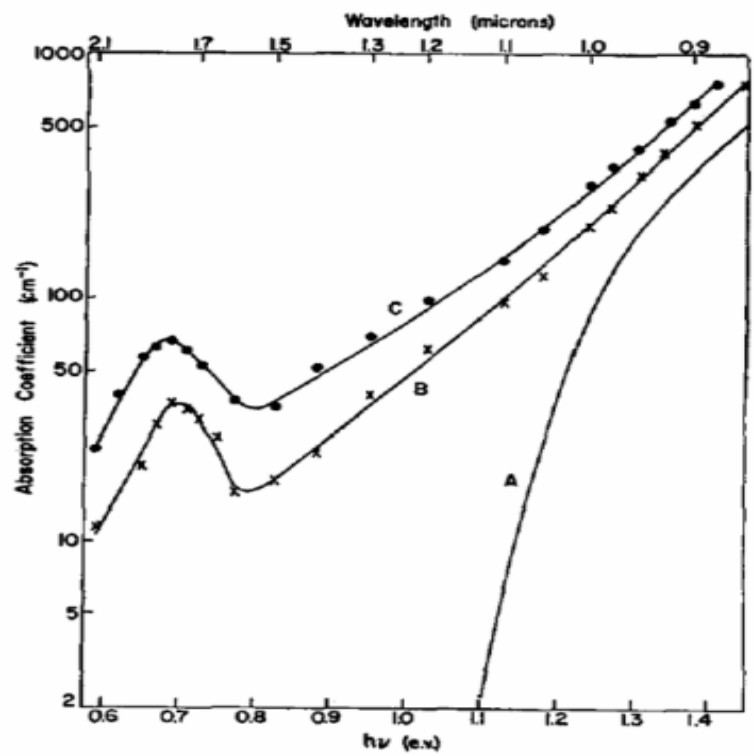

Figure 1. Absorption spectrum of deuteron irradiated Si. A, normal silicon, $\mathrm{B}$ and $\mathrm{C}$ after successive deuteron irradiations. Reprinted with permission from H. Y. Fan and A. K. Ramdas, "Infrared Absorption and Photoconductivity in Irradiated Silicon," Journal of Applied Physics, 30, 1127-1134. Copyright 1959, American Institute of Physics

Two crystal defects are believed responsible for the sub bandgap radiation absorption: divacancies and interstitial cluster. Divacancies show a lower quantum efficiency than the interstitial clusters and and its anneal out between 100 and $300^{\circ} \mathrm{C}$ while the cluster are stable to $>600^{\circ} \mathrm{C}[20,21]$.

\subsection{Surface-State Absorption (SSA)}

The presence of electronic surface states, localized in the forbidden energy gap of a semiconductor, causes an optical absorption extending to energies lower than the edge[22, 23]. In clean surfaces this extra absorption is fairly large and disappears as the surface is slowly oxidized[24]. Allen and Gobeli[25], studying the photoelectric yield modifications of silicon due to the influence of the surface state, showed that their results were in agreement to a surface states two-band model. Garcia-Molinar and Rubio theoretically calculated[26] surface state energy by a known Green's function and, finally, Bortolani et al.[27] by applying this method to the Penn model of a solid (a simplified model) showed the presence of two bands in the gap. The excellent Bortolani's work demonstrated, for firs time, the existence of two band in agreement with the experimental data[25].

Dispersion relations energy as function of momentum $\mathrm{q}$ is reported in Fig. 2(b) in which it is possible to distinguish the bandgap Eg and Fermi momentum $\mathrm{k}_{\mathrm{F}}$. Widths of the bands and gap result in good agreement with optical measurements. States density calculated by Bortolani is reported in Fig. 2(a). Number of states per unit area and band results be $2.5 \times 10^{15}$ states $/ \mathrm{cm}^{2}$, higher than value of $10^{15}$ states $/ \mathrm{cm}^{2}$ given by the Shockley theorem[28].

In 1971 Chiarotti et al.[29] extracted the absorption con- stant as a function of energy by using an experimental set-up in which a beam of monochromatic light was totally reflected, several times, at the surface of a semiconductor cleaved in ultrahigh vacuum (UHV): the intensity of the transmitted light was recorded before oxidizing the surface. The oxidation caused the disappearance of absorbing centers in the surface layer and at this point the transmitted light was measured again.

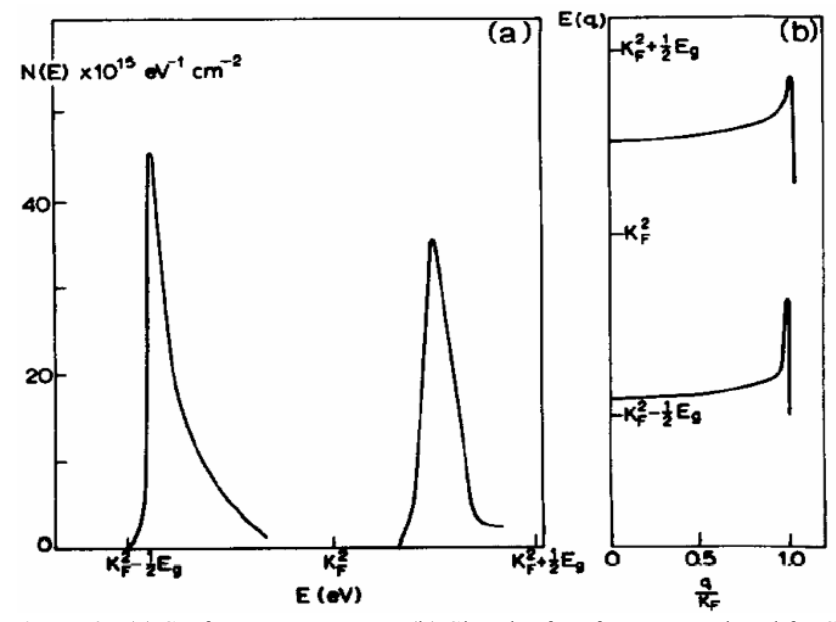

Figure 2. (a) Surface states energy. (b) Sketch of surface energy band for $\mathrm{Si}$ Penn model. Reprinted with permission from Elseiver

The difference between the logarithm of the ratio between the intensities of light impinging on the sample $\mathrm{I}_{0}$ and that emerging from the sample $\mathrm{I}_{\mathrm{T}}$ for a cleaned and oxidized surface of Si allows to extract the superficial absorption constant by the following formula[29]:

$$
\alpha_{s}=\frac{1}{5}\left[\ln \left(I_{0} / I_{T}\right)_{\text {clean }}-\ln \left(I_{0} / I_{T}\right)_{o x}\right]
$$

where the factor $1 / 5$ is introduced to take into account the multiple reflection. In Fig. 3 the absorption constant versus energy, is reported.

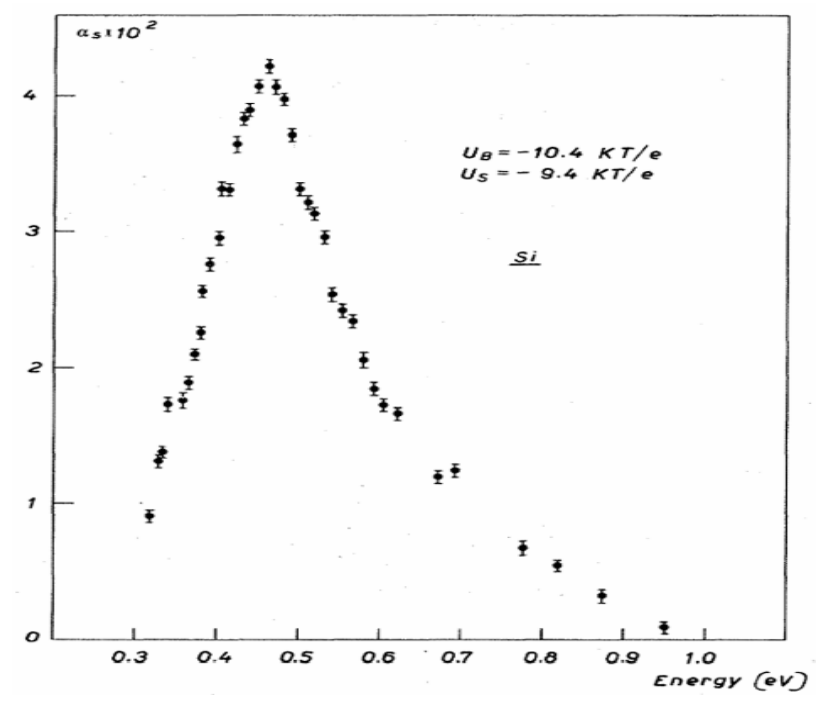

Figure 3. Absorption constant $\alpha$ s vs photon energy for a Si surface. Reprinted with permission from G. Chiarotti et al., "Optical Absorption of Surface States in Ultrahigh Vaccum Cleaved (111) Surfaces of Ge and Si," Physical Review B, 4, 3398-3402, 1971. Copyright 1971 by the American Physical Society 
Different processes could be useful to explain results of Fig. 3: (1) optical transitions between two bands of surface states localized in the gap, (2) optical transitions from the valence band to an empty band of surface states located in the gap and (3) optical transitions from a full band of surface states in the gap to the empty levels of the conduction band. Among these processes the hypothesis (1) seems most probable giving an easier explanation for the peak presence shown in Fig.3[29].

\subsection{Internal Photoemission Absorption (IPA)}

Internal photoemission is the optical excitation of electrons in the metal to energy above the Schottky barrier and then transport of these electrons to the conduction band of the semiconductor (Fig. 4).

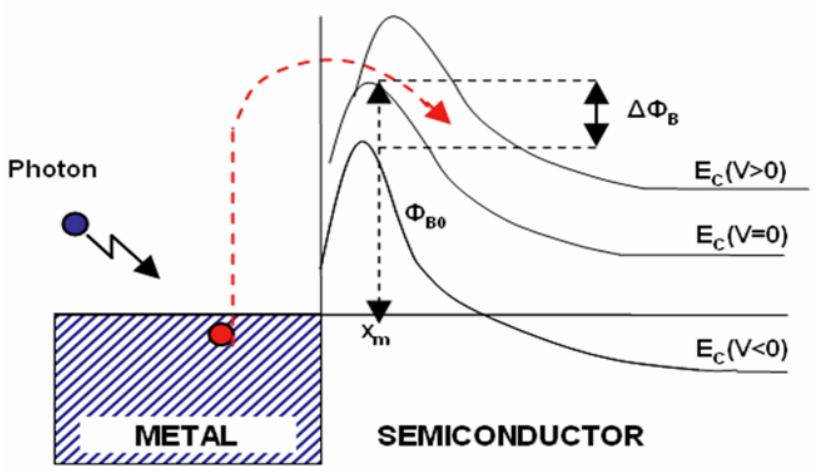

Figure 4. Energy band diagram for a metal $/ \mathrm{n}$-semiconductor junction

The standard theory of photoemission from a metal into a vacuum is due to Fowler[30]. It assumes that the density of states in the metal is described by the normal parabolic distribution associated with nearly-free electron approximation. Thus in Fowler's theory, the Fermi level is several $\mathrm{eV}$ above the conduction band minimum when a single band is considered. In a gas of electrons obeying the Fermi-Dirac statistic, the fraction of the absorbed photons $\mathrm{F}_{\mathrm{e}}$ which produces photoelectrons with the appropriate energy and momentum before scattering to contribute to the photocurrent is calculated as:

$$
F_{e}=\frac{\left[\left(h v-\left(\phi_{B 0}-\Delta \phi_{B}\right)\right)^{2}+\frac{\left(k_{B} T \pi\right)^{2}}{3}-2\left(k_{B} T\right)^{2} e^{\left.-\frac{h v-\left(\phi_{B}-\Delta \phi_{B}\right)}{k_{B} T}\right]}\right.}{8 k_{B} T E_{F} \log \left[1+e^{\left.\frac{h v-\left(\phi_{B}-\Delta \phi_{B}\right)}{k_{B} T}\right]}\right]}
$$

where $\mathrm{h} v$ is photon energy, $\Phi_{\mathrm{B} 0}$ is the potential barrier at zero bias, $\Delta \Phi_{\mathrm{B}}$ is the lowering due to image force effect (as we will see later), $\mathrm{k}$ is Boltzman's constant, $\mathrm{T}$ is the absolute temperature and $\mathrm{E}_{\mathrm{F}}$ is the metal Fermi level.

Fowler's theory was originally obtained without taking into account the thickness of the Schottky metal layer. In order to study the quantum efficiency for thin metal films, the theory must be further extended, taking into account multiple reflections of the excited electrons from the surfaces of the metal film, in addition to collisions with phonons, imperfections and cold electrons.

Assuming a thin metal film, a phenomenological semi- classical ballistic transport model for the effects of the scattering mechanisms resulting in a multiplicative factor for quantum efficiency has been developed by Vickers[31].

The efficiency in collecting those electrons which have sufficient normal kinetic energy to overcome $\Phi_{B}$ will depend on their probability of collisions with cold or hot electrons and with the two boundary surfaces of the metal. Supposing that the Schottky barrier is illuminated through the front surface of the metal electrode, and that optical absorption is uniform throughout the metal thickness, we indicate with $p_{t}$ the average probability that photoexcited electrons arrive at the semiconductor surface without collision. Then, we indicate with $p_{r}$ the accumulated probability of arrival at the reflection boundary of those electrons which have sufficient energy to overcome $\Phi_{\mathrm{B}}$ after one collision with cold or hot electrons, which are oriented with equal probability over the entire half-sphere before reflection by the metal surface.

Finally, for multiple reflections, we indicate with $\mathrm{p}$ the probability that the capturable electrons can bounce from one metal boundary to another. Therefore, the total accumulated probability that the electrons will have sufficient normal kinetic energy to overcome $\Phi_{\mathrm{B}}$ is given by[32]:

$$
P_{E}=p_{t}+p_{r}\left(1+p+p^{2}+p^{3}+\ldots\right) \exp (-d / L) \cong \frac{L}{d}\left[1-e^{-\frac{d}{L}}\right]^{\frac{1}{2}}
$$

where $d$ is the metal thickness and $L$ the mean free path describing the probability of collision with cold or hot electrons[32]. It would be possible to show by plotting Eq. 3 that by decreasing metal thickness $\mathrm{d}, \mathrm{P}_{\mathrm{E}}$ increases, and that a considerable gain can be obtained when $d$ is much smaller than L (thin film). This gain is due to the increased probability of emission of carriers. In a recent work[33], Scales and Berini show that a further enhancement of this probability emission can be obtained in structures realized with thin metal film buried in a semiconductor and forming two Schottky barriers.

In order to estimate the number of electrons which will be able to overcome the potential barrier, two other factors must be taken into account: image force effect and barrier collection efficiency. The image force effect between an electron and the surface of metal causes a potential barrier lowering $\left(\Delta \Phi_{\mathrm{B}}\right)$ and a displacement $\left(\mathrm{x}_{\mathrm{m}}\right)$, as shown in Fig. 4 , which can be calculated with the following formula[34]:

$$
\begin{gathered}
x_{m}=\sqrt{\frac{q}{16 \pi \varepsilon_{S i}} \frac{W}{\left|V_{\text {Bias }}\right|}} \\
\Delta \phi_{B}=\sqrt{\frac{q}{4 \pi \varepsilon_{S i}} \frac{\left|V_{\text {Bias }}\right|}{W}}
\end{gathered}
$$

where $\varepsilon_{\mathrm{Si}}$ is the permittivity of silicon $\left(10^{-12} \mathrm{C} / \mathrm{cmV}\right), \mathrm{W}$ is the depletion width, and $\mathrm{V}_{\text {Bias }}$ the applied bias voltage.

Finally, the probability that an electron travels from the metal-semiconductor interface to the Schottky barrier maximum without scattering in the silicon is taken into account by the barrier collection efficiency $\eta_{c}$, which can be written as[35]: 


$$
\eta_{c}=e^{-\frac{x_{m}}{L_{s}}}
$$

where $L_{s}$ is the electron scattering length in silicon. It is worth noting that by increasing the bias voltage, a shift of the Schottky barrier closer to the metal/semiconductor interface is obtained. In this way, barrier collection efficiency increases.

\subsection{Two-Photon Absorption (TPA)}

In the process of two-photon absorption, an electron absorbs two photons at approximately the same time, achieving an excited state that corresponds to the sum of the energy of the incident photons. In order to reach the final state, no intermediate state is necessary, only a "virtual state" which doesn't correspond to any electronic or vibrational energy eigenstate (Fig. 5).

The absorption cross section $\sigma$ describing this process increases linearly with laser intensity (I) according to the following relation:

$$
\sigma=\sigma^{(2)} I
$$

where $\sigma^{(2)}$ is a coefficient taking into account the probability that the two-photon absorption process occurs. It is clear that in conventional linear optics, the absorption cross section $\sigma$ is a constant. Atomic transition rate $\mathrm{R}$, due to two-photon absorption, scales as the square of the laser intensity, is shown in the following formula:

$$
R=\frac{\sigma^{(2)} I^{2}}{\hbar \omega}
$$

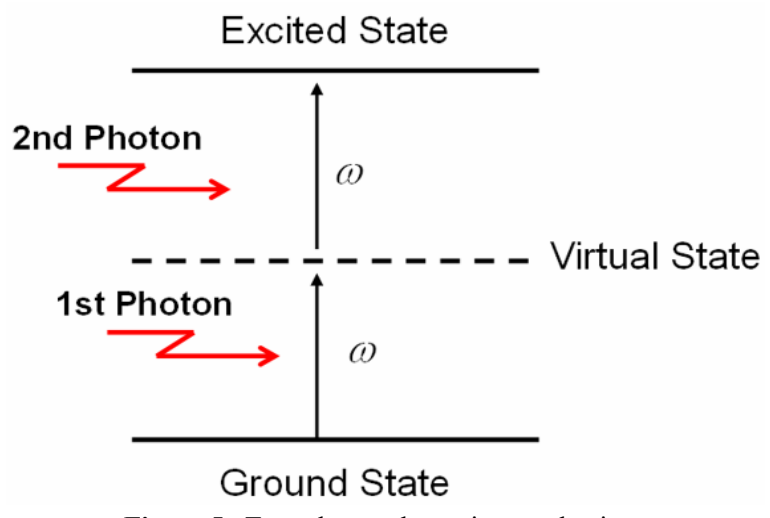

Figure 5. Two-photon absorption mechanism

In the absence of significant recombination and diffusion and without taking into account free-carrier absorption (FCA), the differential equation describing the beam propagation in presence of linear and two-photon absorption can be written as[36]:

$$
d I / d z=-\alpha I-\beta I^{2}
$$

where $\alpha$ is the linear absorption coefficient, $\beta$ is the two-photon absorption coefficient, $\mathrm{z}$ is the longitudinal propagation direction, and where the instantaneous irradiance $I=I(r, z, t)$ is a function of time $t$ and transverse position $r$ as well as z. By assuming $\alpha$ as constant, without taking into account the possible influence of lattice heating, band filling, and bandgap renormalization on $\alpha$, Eq. 8 can be solved ex- actly to give the transmitted irradiance $\mathrm{I}(\mathrm{r}, \mathrm{L}, \mathrm{t})$ in terms of the incident irradiance $\mathrm{I}(\mathrm{r}, 0, \mathrm{t})$ :

$$
I(r, L, t)=T_{0} \frac{I(r, 0, t)}{1+\frac{I(r, 0, t)}{I_{c 2}}}
$$

where:

$$
T_{0}=\left(1-R_{1}\right)\left(1-R_{2}\right) e^{-\alpha L}
$$

is the linear transmission of a sample of thickness $\mathrm{L}$ and front (rear) surface reflectivity $\mathrm{R}_{1}\left(\mathrm{R}_{2}\right)$.

When the irradiance approaches the critical value $I_{\mathrm{c} 2}$ defined as:

$$
I_{c 2}=\frac{(\alpha / \beta)}{\left(1-R_{1}\right)\left(1-e^{-\alpha L}\right)}
$$

the transmission deviates from the linear value and TPA becomes comparable to linear absorption. It is worth noting that when $\mathrm{I}(\mathrm{r}, 0, \mathrm{t})=\alpha / \beta$, the two-photon coefficient near the surface equals the linear coefficient. Of course, $\left(1-\mathrm{R}_{1}\right)$ represents the front surface transmission while $\left(1-\mathrm{e}^{-\alpha \mathrm{L}}\right)$ is the linear absorption of a sample of thickness L. From a physics point of view, $\mathrm{I}(\mathrm{r}, 0, \mathrm{t}) / \mathrm{Ic} 2$ compares the linear absorption coefficient $\alpha$ to the two-photon coefficient $\beta I$.

The quantity typically measured to derive $\beta$ is the transmitted (T) energy, not the irradiance. For a Gaussian spatial and temporal irradiance profile having a maximum $\mathrm{I}_{0}$, it can be shown that[36]:

$$
T^{-1}=T_{0}^{-1}\left[1+\frac{I_{0}}{2 \sqrt{2} I_{c 2}}\right]
$$

It should be noted that this formula has been obtained without taking into account FCA generated by TPA. When FCA cannot be neglected, it can be included, obtaining a modified and more complex formula for the transmittance[36].

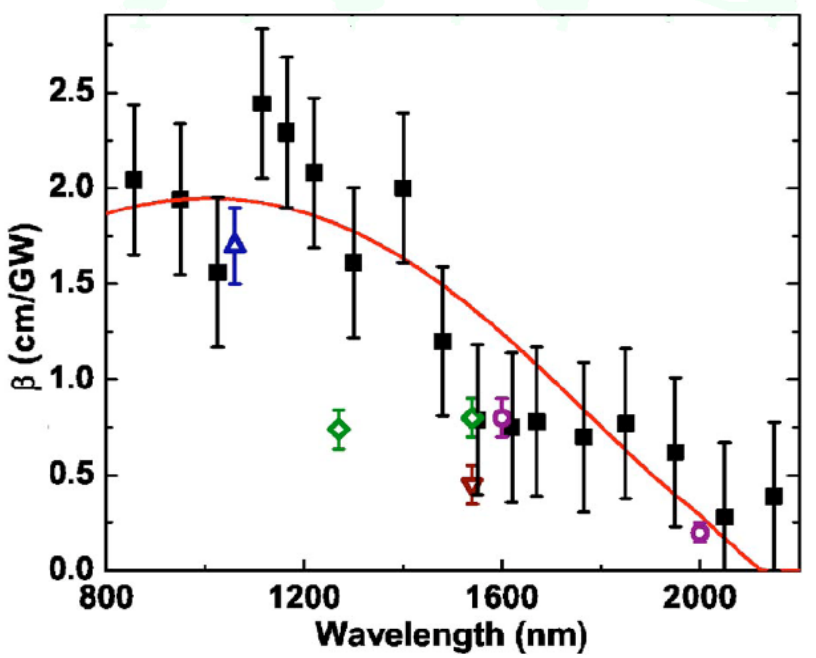

Figure 6. Measured value of $\beta$ (squares) as a function of wavelength for a $125-\mu \mathrm{m}$-thick sample silicon wafer[43]. Data from other sources is also given: circles[44], up triangles[37], down triangles[39], and diamonds[41]. The solid curve represents the best fit based on calculations of Garcia and Kalyanaraman[45]. Reprinted with permission from A. D. Bristow et al. "Two-photon absorption and Kerr coefficients of silicon for 850-2200 nm," Appl. Phys. Lett., 90, 191104. Copyright 2007, American Institute of Physics 
If front and back surface reflectivities are known, it is possible to show that the intercept of an inverse transmission versus irradiance plot corresponds to the linear absorption coefficient, while the initial slope is related to $\beta$.

Reintjes and McGroddy[37] isolated TPA in silicon for first time, obtaining a value of $\beta=1.5 \mathrm{~cm} / \mathrm{GW}$ at $100 \mathrm{~K}$ for picosecond pulses at a wavelength $\lambda=1060 \mathrm{~nm}$, and, in 1986, Boggess et al. measured a similar value at $300 \mathrm{~K}$ [36]. In 1990 , Reitze et al. reported $5<\beta<36 \mathrm{~cm} / \mathrm{GW}$ by measurements across the direct gap of silicon in the range $550<\lambda$ $<620 \mathrm{~nm}$ [38]. In recent years, TPA at telecommunications wavelengths of $\lambda=1500 \mathrm{~nm}$ has been reported, with values of $\beta \sim 0.8 \mathrm{~cm} / \mathrm{GW}$ [39-41]. In 2007, Bristow et al. presented measurements for TPA across the indirect gap $(\mathrm{Eg}=1.12 \mathrm{eV})$ at fundamental wavelengths between 850 and $2200 \mathrm{~nm}$ and compared their results with theoretical predictions[42]. In their work, measurements of $\beta$ are performed using a z-scan technique[43]. Values of $\beta$ as a function of $\lambda$ are illustrated in Fig. 6 . The data is compared with values measured by others.

\section{All-Silicon Photodetector Structures}

In this section, the main NIR silicon photodetector structures will be reviewed, taking advantage of the aforementioned physical effects.

\subsection{MBA-Based Devices}

MBA has been used to develop integrated optical detectors completely compatible with standard silicon technology and sensitive to $\mathrm{C}$ and $\mathrm{L}$ optical band wavelengths. Knights et al., in a work for the Conference on Optical Fiber Communication in 2003, proposed a monolithic p-i-n waveguide photodiode based on implantation into a silicon-on-insulator wafer[46]. In particular, the implantation of protons into a rib waveguide with a lateral p-i-n diode (Fig. 7) was used to create the deep levels. Thus, defects could be controlled in terms of concentration, depth and location via selective masking. On the other hand, shallow implantation and rapid thermal annealing of the $\mathrm{p}+$ and $\mathrm{n}+$ contact regions allowed for the separation of the contacts to be close to the width of the waveguide without introducing excessive free carrier absorption. The proposed 10-mm-long photodiode exhibited a responsivity of about $8 \mathrm{~mA} / \mathrm{W}$ at $1550 \mathrm{~nm}$ and a leakage current of about $3 \mathrm{nA}$, in both cases with $1 \mathrm{~V}$ of applied reverse bias.

Bradley, Jessop, and Knights have investigated different factors useful for improving both the performance and repeatability of integrated photodiodes[47,48]. Due to the high deep trap density, carriers are characterized by a lifetime in the nanosecond range and thus by a diffusion length of the order of a few microns.

This implies a dependence of device performance on the separation between the waveguide, where the carriers are generated, and the semiconductor p-n junction[38]. Moreover, in 2006, Knights et al.[48] demonstrated the importance of post-implantation annealing on device performance.

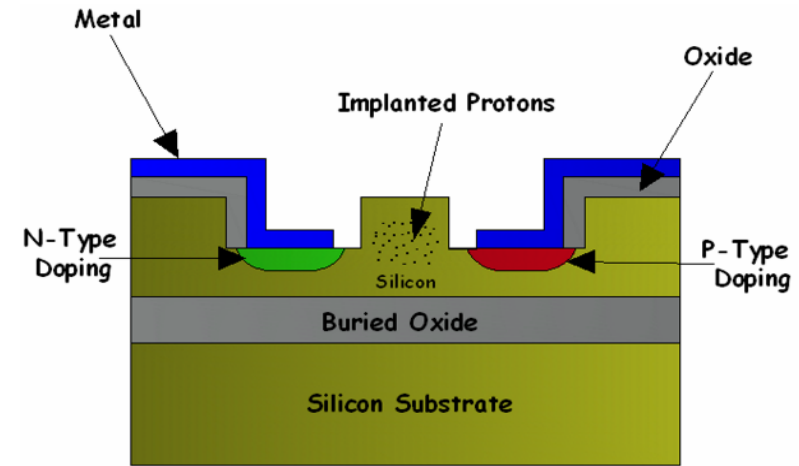

Figure 7. Monolithic p-i-n waveguide photodiode proposed by Knights et al.[46] in 2003

Fig. $8 \mathrm{a}$ and $8 \mathrm{~b}$ report plots derived by the authors with regards to the dependence of the propagation optical losses and the photocurrent generated on the post-implantation annealing temperature for an integrated photodetector with defects introduced by silicon-ion bombardment. The plots demonstrate that an improvement of device performance can be obtained through low-temperature annealing (around $300^{\circ} \mathrm{C}$ ). For these temperatures, carrier recombination centers that do not contribute to the carrier generation process are removed. This should imply a reduction of the competing mechanisms of carrier generation and thus a significant improvement both of the photoresponse and optical loss within the waveguide.

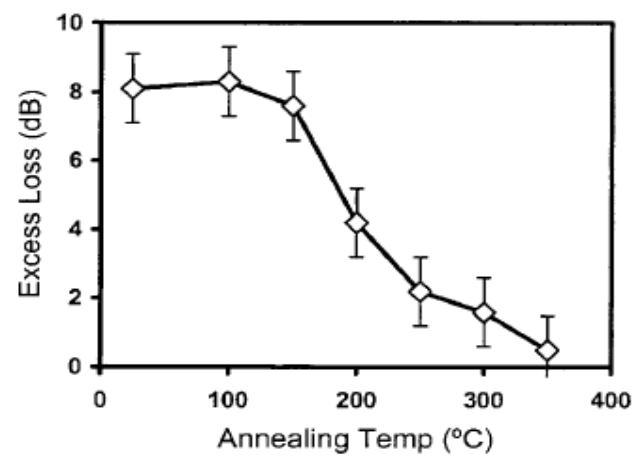

(a)

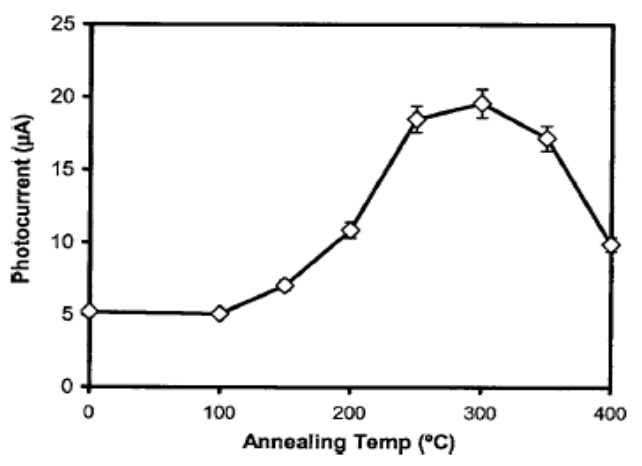

(b)

Figure 8. Optical loss (a) and photocurrent (b) vs post-implantation annealing temperature for an ion-implanted integrated photodiode[48]. Reprinted with permission from J. Vac. Sci. Technol. A24, 783 (2006). Copyright 2006 American Vacuum Society

Different implanted ions were used by Liu et al.[49]; in 
particular, helium ions were chosen for their non-reactivity with silicon and for stability of created defects[50]. Liu et al. carried out a detailed analysis of the influences of ion- implantation doses and of annealing temperature, both on the photogenerated current and the optical losses. This analysis led to the fabrication of an optimized integrated photodetector that was $1.7 \mathrm{~cm}$ long, and which obtained a maximum value of responsivity of $64 \mathrm{~mA} / \mathrm{W}$ at $1440 \mathrm{~nm}$ and a leakage current of $0.1 \mu \mathrm{A}$, both with a reverse bias of $20 \mathrm{~V}$.

All the described photodetectors have been characterized by a very large traversal section in the order of tens of $\mu \mathrm{m}^{2}$, and by absorption lengths in the $\mathrm{cm}$ range. Thus, the bandwidth of these devices was rather limited. Reducing the waveguide dimensions and optimizing the implantation in the silicon waveguide, Geis et al.[51] demonstrated the feasibility of robust high-frequency integrated photodiodes. The main concept is related to the decrease of the transversal cross section of the waveguide. In fact, this scaling induces a larger overlap between the optical mode and the implanted region and thus increases optical absorption, reducing the required device length and increasing the frequency response.

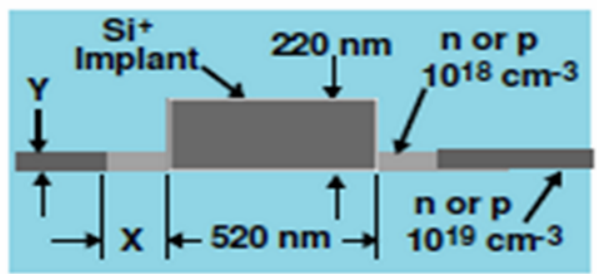

(a)

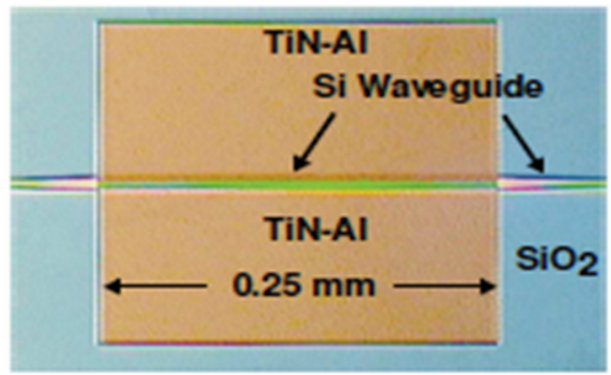

(b)

Figure 9. Top-view optical micrograph (a) and schematic cross section (b) of the integrated photodiode proposed by Geis et al.[52]. Reprinted with permission from M. W. Geis et al., "Silicon waveguide infrared photodiodes with $>35 \mathrm{GHz}$ bandwidth and phototransistors with $50 \mathrm{AW}-1$ response," Opt. Express, 17, 5193-5204, 2009. Copyright 2009 by Optical Society of America

After a rigorous process of optimization[51, 20], the photodetector proposed by Geis et al. had a cross section of about $0.11 \mu \mathrm{m}^{2}$ showing an internal responsivity of $0.5-0.8$ $\mathrm{A} / \mathrm{W}$ at $1550 \mathrm{~nm}$, a reverse bias of about $5 \mathrm{~V}$, a leakage current of $2.5 \mathrm{nA} / \mathrm{mm}$ and a bandwidth of 10-20 GHz. Further, to increase the device bandwidth, Geis and his co-authors optimized the external circuit components[52] such as the resistance and capacitance of the silicon wings on the sides of the rib wavelength (Fig. 9) and on the contact pads.

This optimization allowed minimizing the effect of the parasitic components and thus increased the detector bandwidth above $50 \mathrm{GHz}$. Unfortunately, Geis's devices have low optical absorption, requiring a diode on the order of millimetres in length to absorb more than $50 \%$ of the incoming light. Recently, for the OFC/NFOEC 2010 conference in San Diego, Shafiiha et al. proposed a drastic reduction of the device size using a resonance structure[53]; in particular, the light from a straight waveguide coupled into a small-defect $\mathrm{Si}+$ implanted ring resonator (Fig. 10).

The ring resonator [54-56] was developed in order to equal the power coupling between the ring and the bus waveguide and the round-trip loss of the ring resonator at the resonance wavelength. In this condition, most of the transmission light is absorbed, increasing electron-hole generation. In Figure 10a, the transverse section of the ring waveguide is sketched, and the overlap between the region of the optical mode, the defect area obtained and the depletion region of the p-i-n diode is illustrated. In Figure 10b, a SEM image of the $15-\mu \mathrm{m}$-radius-ring integrated photodetector is reported. The photocurrent of the ring detector under a reverse bias of $-2 \mathrm{~V}$ is illustrated in Fig. 11.

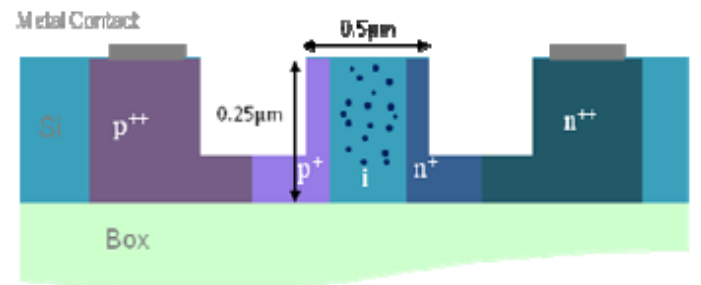

(a)

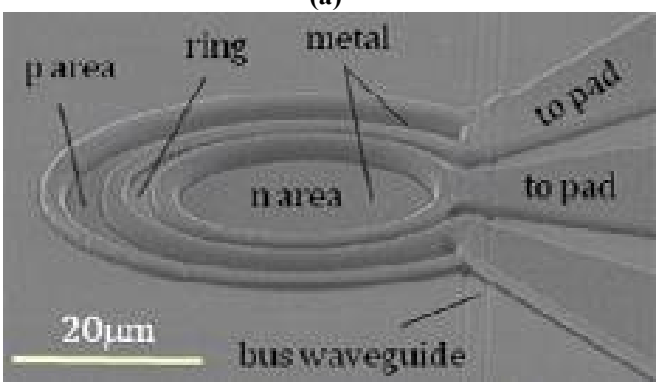

(b)

Figure 10. Cross section (a) and top view SEM image (b) of the fabricated ring detector[53]. Reprinted with permission from R. Shafiiha et al., "Silicon waveguide coupled resonator infrared detector," In Proceedings of Optical Fiber Communication Conference, San Diego, CA, USA, March 21-25, 2010. Copyright 2010 by Optical Society of America

In particular, photocurrents for both before and after a burn in process are reported. The burning-in process, that was also used by Geis et al.[20], consists of a forward bias with a current density of $300 \mathrm{~mA} / \mathrm{cm}$ for 5 minutes and makes possible an increase in the responsivity of the ring photodetector from $0.02 \mathrm{~A} / \mathrm{W}$ up to about $0.1 \mathrm{~A} / \mathrm{W}$ at 1549 $\mathrm{nm}$ and $-2 \mathrm{~V}$. Reported leakage current is $0.1 \mathrm{nA}$ at $-2 \mathrm{~V}$ of applied reverse bias.

The compactness of the device allows reaching a 3-dB bandwidth of about $7 \mathrm{GHz}$ at $-2 \mathrm{~V}$ reverse bias. However, all the reported performances of the ring photodetector are relative to the resonance wavelength and thus the $\mathrm{C}+\mathrm{L}$ bands 
are not completely covered.

To overcome this limitation, Doylend et al. recently proposed a new ring-resonator-based photodetector[57]. The device was characterized by a responsivity of $0.14 \mathrm{~A} / \mathrm{W}$ with a reverse bias of $-10 \mathrm{~V}$ and a leakage current of $0.2 \mathrm{nA}$; i.e., performance that is comparable with that of Shafiiha's device. However, the novelty of the proposed structure is the possibility of tuning the photodetector response in a wavelength range from $1510 \mathrm{~nm}$ to $1600 \mathrm{~nm}$. In particular, resonance wavelength shift has been obtained by thermo-optic tuning of the ring resonance, using a resistive metal strip which overlaps the structure.

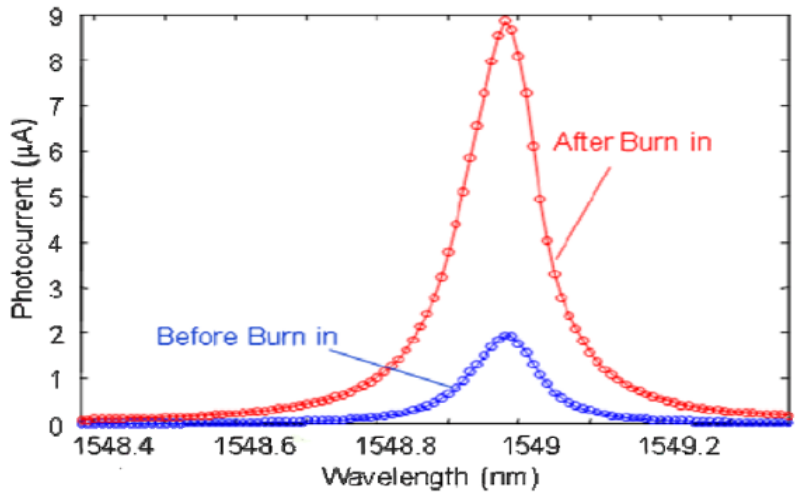

Figure 11. Photoresponse of a $15-\mu \mathrm{m}$-radius-ring detector at $-2 \mathrm{~V}[53]$. Reprinted with permission from R. Shafiiha et al., "Silicon waveguide coupled resonator infrared detector," In Proceedings of Optical Fiber Communication Conference, San Diego, CA, USA, March 21-25, 2010. Copyright 2010 by Optical Society of America

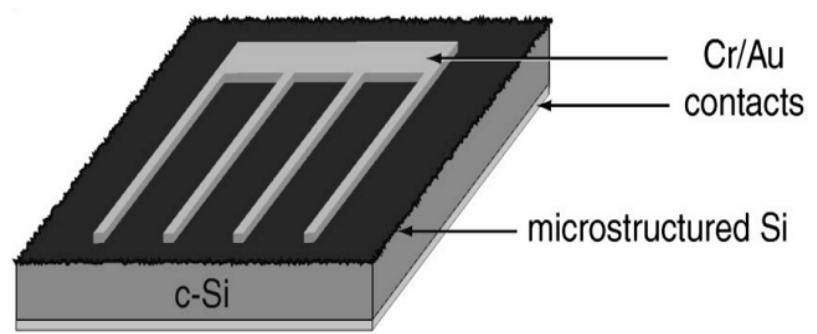

Figure 12. Bulk photodiode proposed by Carey et al.[59] in 2005. Reprinted with permission from J. E. Carey et al., "Visible and near-infrared responsivity of femtosecond-laser microstructured silicon photodiodes," Opt. Lett., 30, 1773-1775, 2005. Copyright 2005 by Optical Society of America

Although MBA has been well-known for quite some time, very few papers concerning silicon-based bulk devices have been reported in the literature, and yet several integrated devices are fabricated and characterized. In $2001, \mathrm{Wu}$ et al.[58] observed a remarkable increase of IR absorbance in surface microstructured silicon wafers produced by laser irradiation in the presence of $\mathrm{SF}_{6}$. They found that in the microstructuring process, a high concentration of impurities and structural defects were incorporated into the silicon lattice, most likely producing bands of impurity states in the band gap that could absorb infrared radiation. The ion channelling spectra obtained from microstructured wafer show a background level higher than that of crystalline silicon but lower than that of amorphous silicon. These measurements indicate that the material is crystalline and is likely to have a high density of defects. The background remains high in ion channelling spectra from samples annealed for 3 hours at $1200 \mathrm{~K}$, indicating that a significant amount of disorder in the microstructured material is unaffected by annealing under these conditions. Moreover, the resulting subgap absorption is enhanced by the surface texture. These remarkable properties were then exploited for the fabrication of a silicon-based detector for infrared radiation[59]. The diode characteristics and responsivity depend strongly on processing conditions including laser fluence, substrate doping, and thermal annealing temperature. Fig. 12 shows a schematic diagram of the device, whose active area is approximately $5 \mathrm{~mm}^{2}$.

This optimized sample exhibits responsivity of $50 \mathrm{~mA} / \mathrm{W}$ at $1330 \mathrm{~nm}$ and $35 \mathrm{~mA} / \mathrm{W}$ at $1550 \mathrm{~nm}$ at $0.5 \mathrm{~V}$ of applied reverse bias; its capacitance is $64 \mathrm{nF} / \mathrm{cm}^{2}$, while the signal rise time is $10 \mathrm{~ns}$ and the fall time is $30 \mathrm{~ns}$. Device leakage current is $120 \mu \mathrm{A} / \mathrm{cm}^{2}$ at $0.5 \mathrm{~V}$ of applied reverse bias.

\subsection{SSA-Based Devices}

In 2007, Geis et al.[51] observed a low photoresponse for an unimplanted diode and attributed it to a surface effect of the waveguide even if its performances were a strong function of the operating environment (such as humidity and surface chemistry). Baehr-Jones et al.[60] enhanced the effect of the surface states optimizing the overlap between the optical mode and the surface of the waveguide. The SEM image and a top view of one of the devices characterized by the authors are shown in Fig. 13a and 13b, respectively.

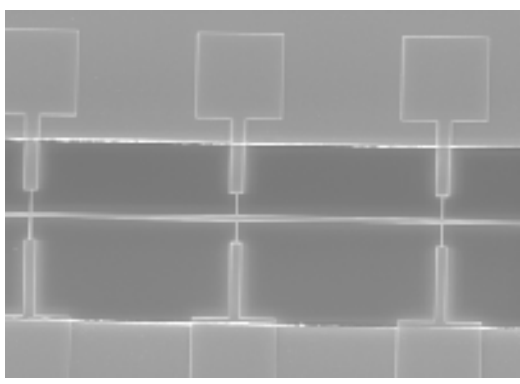

(a)

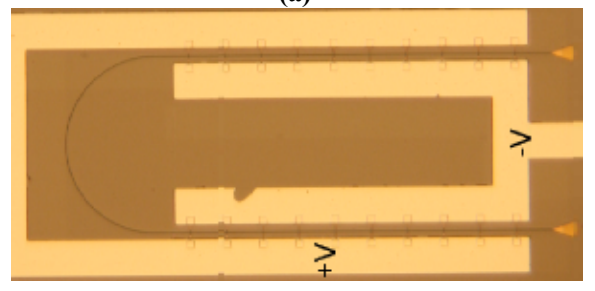

(b)

Figure 13. Top view SEM image (a) and optical image (b) of the detector proposed by Baehr-Jones et al.[60]. Reprinted with permission from Baehr-Jones et al., "Photodetection in silicon beyond the band edge with surface states," Opt. Express, 16, 1659-1668, 2008. Copyright 2008 by Optical Society of America

Small conductions arms intersects the waveguide defining the active regions of the photodetector. The waveguide was developed with a cross section of $500 \mathrm{~nm} \times 100 \mathrm{~nm}$ in order to increase overlap between the optical mode and the waveguide surface. In this way, part of the optical mode is 
absorbed by the surface states and a change in conductivity of the device is achieved. For the device illustrated in Figure 13 , a responsivity of $36 \mathrm{~mA} / \mathrm{W}$ for a bias of $11 \mathrm{~V}$ at a wavelength of $1575 \mathrm{~nm}$ was measured. A device leakage current of $0.12 \mu \mathrm{A}$ at $-11 \mathrm{~V}$ is reported as well. Moreover, the authors reported a detailed analysis to demonstrate that the measured photocurrent is due to the energy state of the surface defects and not to other effects such as heating, two-photon absorption or internal photoemission.

In order to enhance the photocurrent generated by SSA, Chen et al.[61] used a p-i-n diode embedded into a silicon micro-ring resonator. Figure 14a shows a top view of Chen's proposed device; whereas in Figure 14b, the waveguide dimension obtained on a SOI wafer with a $1-\mu \mathrm{m}$-thick buried oxide layer is illustrated. A low-temperature deposited oxide layer with a thickness of $1 \mu \mathrm{m}$ has been used to cover the waveguide. In Figure 14b, the intensity contour of the simulated TE optical mode field is reported and the spatial overlapping with the $\mathrm{Si} / \mathrm{SiO}_{2}$ interfaces can be noted. This overlapping makes possible achievement of photogenerated current by SSA. The ring resonator was characterized by a Q factor of about 8000 . With this value, the responsivity detected at the resonance wavelength $(1541.5 \mathrm{~nm})$ for $0 \mathrm{~V}$ bias was about $0.12 \mathrm{~mA} / \mathrm{W}$ and resulted twenty times that of the responsivity measured for off-resonance wavelengths. Increasing the bias voltage to $-15 \mathrm{~V}$, the responsivity increased to $0.25 \mathrm{~mA} / \mathrm{W}$ but the dark current reached $2.5 \mathrm{nA}$.

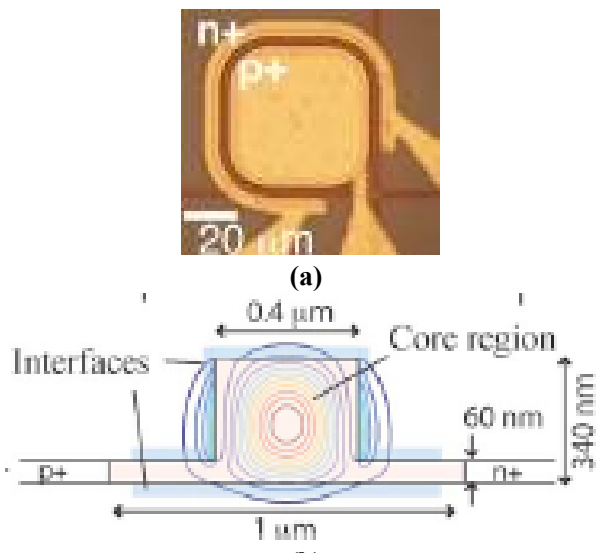

(b)

Figure 14. (a) Top view of the ring resonator detector. (b) Sketch of the device and contour of optical mode[61]. Reprinted with permission from $\mathrm{H}$. Chen et al., "Cavity-enhanced photocurrent generation by $1.55 \mu \mathrm{m}$ wavelengths linear absorption in a p-i-n diode embedded silicon microring resonator," Appl. Phys. Lett., 95, 171111. Copyright 2009, American Institute of Physics

Due to the presence of a resonator, the device became more sensitive to temperature changes. In particular, the authors measured a redshift of the resonance wavelength that was explained by the thermo-optical effect and the heating due to the carrier recombination. Finally, the authors noted a slight linear absorption-induced photovoltaic effect, with a power generation efficiency of about $0.05 \mathrm{~mW} / \mathrm{W}$.

\subsection{IPA-Based Devices}

In this section, the main NIR silicon photodetectors structures based on the afore-mentioned IPE effect will be reviewed. Silicon infrared photodiodes based on IPE are not novel, in fact $\mathrm{PtSi} / \mathrm{p}-\mathrm{Si}, \mathrm{Ir} / \mathrm{p}-\mathrm{Si}$ and $\mathrm{Pd}_{2} \mathrm{Si} / \mathrm{p}$-Si junctions are usually used in the infrared imaging systems[62]. The main advantages of these devices resides in their extremely high switching speed and in their simple fabrication process, but, due to high background current density these devices can only work at cryogenic temperature. However, in recent years IPE has emerged as a new option for detecting also near infrared (NIR) wavelengths at room temperature[63-65]. Unfortunately, the photoemission quantum efficiency is low compared to that of detectors based on inter-band absorption and this limits the application both in power monitoring and in the telecommunication field. Low quantum efficiency is a direct result of conservation of momentum during carrier emission over the potential barrier, in fact, the majority of excited carriers which do not have enough momentum normal to the barrier are reflected and not emitted. Moreover as incoming photons can excite carriers lying in states far below the Fermi energy, which can not overcome over the metal-semiconductor potential barrier, the quantum efficiency of these devices is further decreased. In recent years new approaches and structures are proposed in order to circumvent these limitations and new designs have been proposed for achieving a suitable quantum efficiency. Elabd et al. proposed to use a very thin metal film (about $2 \mathrm{~nm}$ ) to increase the escape probability of hot carriers due to their multiple reflections inside the metal film[66]. In particular, the proposed Schottky photodetector was characterized by a responsivity of about $250 \mathrm{~mA} / \mathrm{W}$ for a wavelength $\lambda=1500$ $\mathrm{nm}$. However, since the volume in which the photons interact with electrons in the metal is very small, only a small fraction of the incident photons actually causes photoemission. Several solutions have been proposed to enhance the efficiency of the IPE process. For example, in the 2001 Wang's group[67] investigated the spectral responsivity of Al-porous silicon Schottky barrier photodetectors in the wavelength range 0.4-1.7 $\mu \mathrm{m}$. The structure of the PS photodetector was $\mathrm{Al}$ (finger type)/PS/Si/Al (ohmic), and the active area was 18 $\mathrm{mm}^{2}$. The photodetectors show strong photoresponsivity in both the visible and the infrared bands, especially at $1.55 \mu \mathrm{m}$. The photocurrent can reach $1.8 \mathrm{~mA}$ at a reverse bias of $6 \mathrm{~V}$ under illumination by a $1.55-\mu \mathrm{m}, 10-\mathrm{mW}$ laser diode. The corresponding quantum efficiency is $14.4 \%$; this high value comes from a very high surface-area-to-volume ratio, of the order of $200-800 \mathrm{~m}^{2} / \mathrm{cm}^{3}$ of porous Si. The dark current is $\sim 5$ $\mu \mathrm{A}$ at $-10 \mathrm{~V}$. Recently, Casalino et al.[68, 69] propose to enhance the IPE absorption by a resonant cavity effect. The conceptual scheme of the proposed device in shown in Fig. 15.

The resonant cavity is a vertical-to-the-surface FabryPerot structure. It is formed by a buried reflector, a top mirror interface and, in the middle, a silicon cavity. The buried reflector is a Bragg mirror, realized by alternating layers of amorphous hydrogenated silicon $(\mathrm{a}-\mathrm{Si}: \mathrm{H})$ and silicon nitride $\left(\mathrm{Si}_{3} \mathrm{~N}_{4}\right)$. A Schottky metal layer $(\mathrm{Cu})$, working both as active (absorbing) layer and as cavity mirror, is deposited above the 
silicon layer. The room temperature responsivity measurements on the device return a peak value of about $2.3 \mu \mathrm{A} / \mathrm{W}$ and $4.3 \mu \mathrm{A} / \mathrm{W}$, respectively for $0 \mathrm{~V}$ and $-10 \mathrm{mV}$ of reverse bias applied[68]. In[69] the authors propose both to scaling down and optimize the same device described in[68]. In particular, the cavity finesse was increased allowing to reach a responsivity of $8 \mu \mathrm{A} / \mathrm{W}$ for a reverse bias of $100 \mathrm{mV}$.

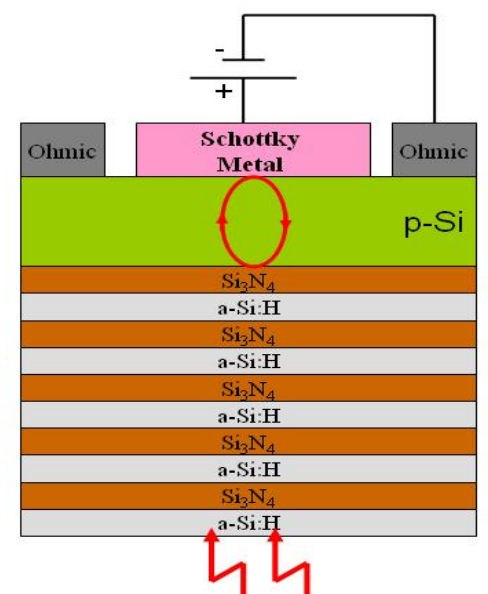

Figure 15. Schematic cross section of the photodetector proposed by Casalino et al.[68,69]

A substantial enhancement of the IPE process efficiency has been achieved increasing the interaction of light with the metal in the vicinity of the interface by the confinement of the infrared radiation into a silicon waveguide. This solution has effectively proven that Schottky diode photodetectors are good candidate for the highly integrated photonics circuits. An example of this approach has recently demonstrated by Coppola's group[70].

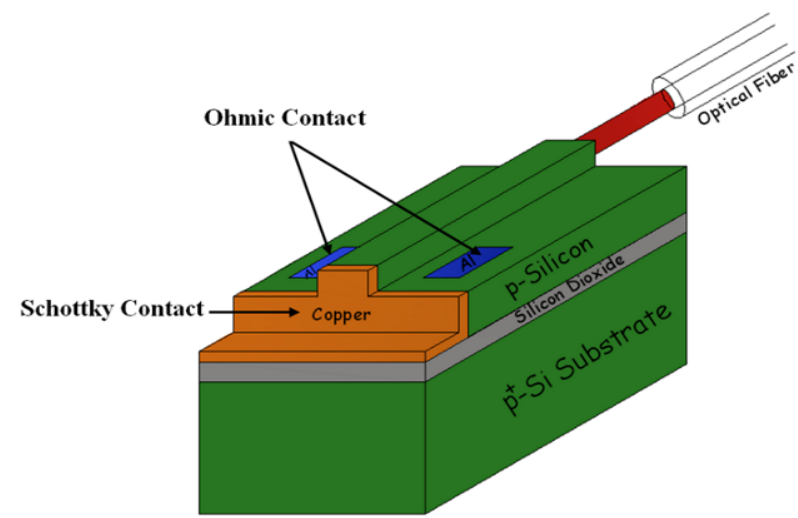

Figure 16. Schematic view of the $\mathrm{Cu} / \mathrm{p}$-Si Schottky barrier-based integrated photodetector proposed by Casalino et al.[70]. Reprinted with permission from M. Casalino et al., "Cu/p-Si Schottky barrier-based near infrared photodetector integrated with a silicon-on-insulator waveguide," Appl. Phys. Lett., 96, 241112. Copyright 2010, American Institute of Physics

The proposed device is schematically illustrated in Fig. 16. A rib waveguide was terminated on a deep trench that reaches down the buried oxide layer of the SOI wafer. A $\mathrm{Cu} / \mathrm{p}-\mathrm{Si}$ Schottky contact was fabricated on the vertical surface of the deep trench.

By means of this technological solution a very narrow semiconductor/metal barrier transverse to the optical field coming out from the waveguide has been achieved. The integrated photodetector was characterized by a responsivity of $0.08 \mathrm{~mA} / \mathrm{W}$ at a wavelength of $1550 \mathrm{~nm}$ with an reverse bias of $-1 \mathrm{~V}$. Measured dark current at $-1 \mathrm{~V}$ was about $10 \mathrm{nA}$. Moreover, the authors assert that the thinness of $\mathrm{Cu} / \mathrm{p}-\mathrm{Si}$ Schottky barrier could enable a speed operation in the gigahertz range.

An indirect evaluation of the bandwidth of the detector was reported to confirm the operation speed potentialities. A bandwidth of about $3 \mathrm{GHz}$ was measured by Zhu et al. in[71] on a Schottky barrier based integrated photodetector, where the junction was achieved by a nickel silicide layer $\left(\mathrm{NiSi}_{2}\right)$ on silicon[72, 73](Fig. 17).

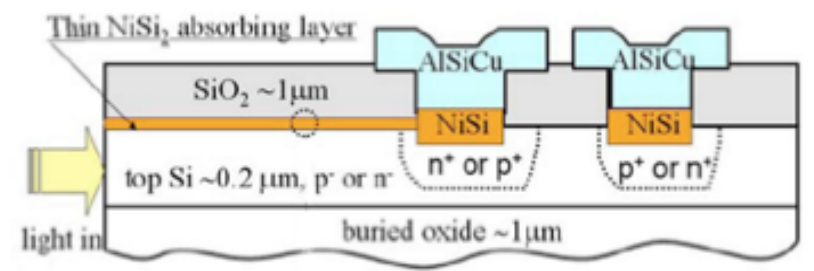

Figure 17. Schematic structure of waveguide-based silicide Schottkybarrier photodetector proposed by Zhu et al.[72, 73]. Reprinted with permission from S. Zhu et al., "Low-cost and high-gain silicide Schottky- barrier collector phototransistor integrated on Si waveguide for infrared detection," Appl. Phys. Lett., 93, 071108. Copyright 2008, American Institute of Physics

In particular, the authors proposed the lengthening of a thin silicide layer on the surface of a SOI waveguide to achieve both a suitable optical absorption and an efficient photoexcitation of metal electrons or holes across the silicide/Si interface. A detailed analysis was reported on the influence of the silicide layer dimension on the performances of both $\mathrm{NiSi}_{2} / \mathrm{p}-\mathrm{Si}$ and $\mathrm{NiSi}_{2} / \mathrm{n}$-Si diodes. The overall performances of the $\mathrm{NiSi}_{2} / \mathrm{p}$-Si structure have been resulted better than that relative to the $\mathrm{NiSi}_{2} / \mathrm{n}-\mathrm{Si}$ interface, due to the lower Schottky barrier height of the latter structure. In particular, a responsivity of about $4.6 \mathrm{~mA} / \mathrm{W}$ at a wavelength of $1550 \mathrm{~nm}$ and a reverse bias of $-1 \mathrm{~V}$ was estimated for the $\mathrm{NiSi}_{2} / \mathrm{p}$-Si diode against the value of $2.3 \mathrm{~mA} / \mathrm{W}$ of the $\mathrm{NiSi}_{2} / \mathrm{n}$-Si junction. Moreover, also the $3 \mathrm{nA}$ of the measured dark current can be considered acceptable.

In order to increase further the capability of IPE-based silicon photodetector to detect long-wavelength (infrared) photons, the possibility to use the concept of surface plasmon polaritons (SPPs) has been explored. SPP are TM-polarized electromagnetic waves trapped at or guided along a metaldielectric interface. This effect was discovered several decades ago but attention has been renewed by the phenomena of enhanced optical transmission through metallic films with nanostructure[74,75]. In fact, SPPs are shorter in wavelength than the incident light providing a significant increase in spatial confinement and local field intensity. In other words, while optical systems are basically diffraction limited, surface plasmon polaritons allow a tight localization of optical field to strongly subwavelength dimensions at the metaldielectric interface[76,77]. Such property means that the infrared light is directly guided toward the active area of the 
IPE detector, confining the optical power at the boundary between the materials forming the Schottky contact, thereby increasing the interaction of light with the metal in the vicinity of the interface where the photoemission process takes place. Berini's group at University of Ottawa has recently applied these concepts confirming that the high light confinement of the SPPs structures can significantly improve the detection capability of the IPE-based photodetector integrated on Si wafers. In particular, Scales et al. in[78,79] described a Schottky diode photodetector obtained embedding a metal stripe of finite width in a homogeneous dielectric cladding (symmetric structure). The proposed device (see Fig. 18) was characterized by a responsivity of about 0.1 $\mathrm{A} / \mathrm{W}$, a dark currents of $21 \mathrm{nA}$, and minimum detectable powers of $-22 \mathrm{dBm}$ at a wavelength $\lambda=1550 \mathrm{~nm}$.

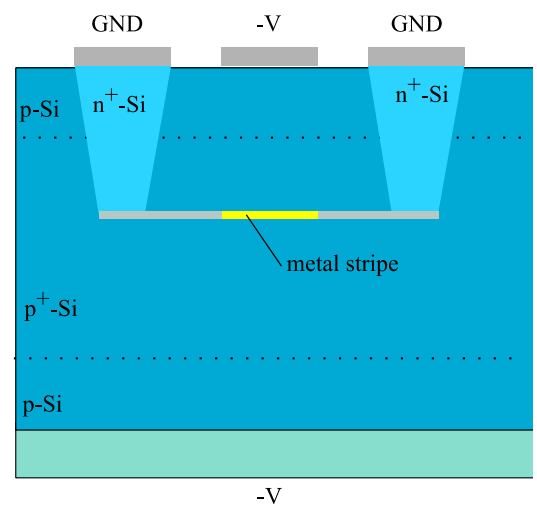

Figure 18. SPPs Schottky detector based on a metal stripe surrounded by silicon

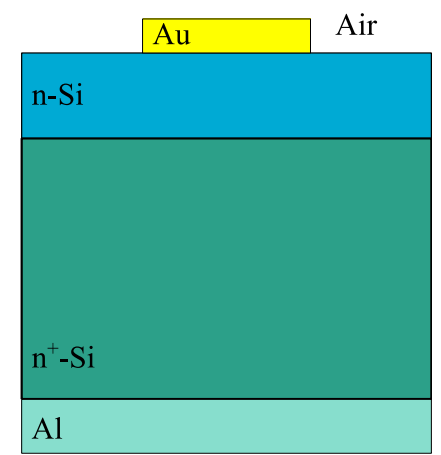

Figure 19. Cross-section of the aymmetric SPPs Schottky detector proposed in (Akbari et al., 2010)

An accurate investigation of the performance of the same device for different wavelengths and for several metals forming Schottky contacts were carried out in[80]. It is worth noting that in these symmetric structures the realization of a thin metal film buried in a semiconductor become the fabrication process more complicated, however, despite of this drawback, the IPE is enhanced due to emission carriers occurring through two Schottky barriers[33]. In order to simplify the fabrication process and to obtain a very short device, Berini's group has proposed an asymmetric SPPs-based photodetector[81,82]. The proposed structure supports highly confined and highly attenuated SPP modes; this latter feature allows to fabricate shorter devices compared to the symmetric photodetectors. The device, sketched in Fig. 19, consists of a metal stripe cladded at the bottom by a layer of Silicon and covered by air and exhibits for a wavelength $\lambda=1280 \mathrm{~nm}$ a maximum value of responsivity of about 1 $\mathrm{mA} / \mathrm{W}$ with a dark current of $6 \mu \mathrm{A}[82]$.

A detailed simulation analysis performed on asymmetric Schottky detector shows that a significant enhancement in the responsivity can be achieved for a thin metal stripe (about $5 \mathrm{~nm}$ ) due to multiple internal reflections of excited carriers[81]. In particular, authors report that the enhancement is more noticeable for thin metal stripe on $\mathrm{p}$-Si compared to device on $\mathrm{n}$-Si. In this case, the energy range over which the hot electrons can experience multiple reflections is very small because of the larger Schottky barrier height. Finally, the same group has recently demonstrated that a considerable increase in responsivity can be reached with strong applied reverse bias[83]. In particular, authors driving a no optimized detector like that shown in Fig. 19 into breakdown $(\mathrm{V} \sim-210 \mathrm{~V})$, such that internal electronic gain is obtained by carrier multiplication, obtaining a responsivity of 2.35 $\mathrm{mA} / \mathrm{W}$.

As mentioned above the possibility to achieve a strong light confinement implies both an improvement of responsivity and an important advancement in device miniaturization enabling the realization of on-chip photodetectors on the nanoscale. In this contest, Goykhman et al. in[84] have characterized a nanoscale silicon surface-plasmon Schottky detector shown in Fig. 20.

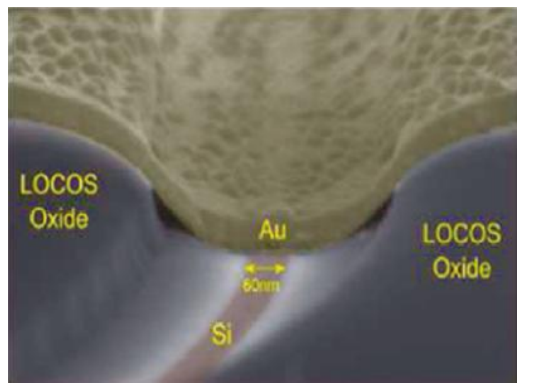

Figure 20. SEM micrograph of the nanoscale Schottky contact proposed in[84]. Reprinted with permission from I. Goykhman et al., "Locally Oxidized Silicon Surface-Plasmon Schottky Detector for Telecom Regime," Nano Lett., 11, 2219-2224, 2011. Copyright 2011 by American Chemical Society

The detector was fabricated employing a self-aligned approach of local-oxidation of silicon (LOCOS) on silicon on insulator substrate. This approach has been proved useful for fabricating in the same process both a low-loss bus photonic waveguide and the detector. Actually, the oxide spacers effectively define the nanometric area of metal-silicon interface and thus allow avoiding lateral misalignment between the silicon surface and the metal layer to form a Schottky contact. The so realized photodetector was characterized by a responsivity $0.25,1.4$, and $13.3 \mathrm{~mA} / \mathrm{W}$ for incident optical wavelengths of $1.55,1.47$, and $1.31 \mu \mathrm{m}$, respectively.

\subsection{TPA-Based Devices}

Despite the small value of the TPA coefficient, ranging from $0.44 \mathrm{~cm} / \mathrm{GW}$ to $0.9 \mathrm{~cm} / \mathrm{GW}$ at a wavelength of 1550 
$\mathrm{nm}[85]$, silicon optical waveguides have potential for use in TPA because of their long interaction lengths and low optical dispersion in silicon. The photocurrent generated by the TPA effect can efficiently collect embedding a $\mathrm{p}-\mathrm{i}-\mathrm{n}$ diode in the silicon waveguide. In Fig. 21a, the TPA-based integrated detector proposed by Liang et al. in 2002 is reported[86].

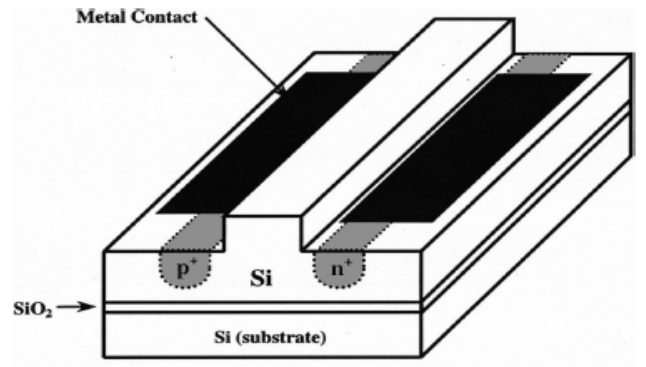

(a)

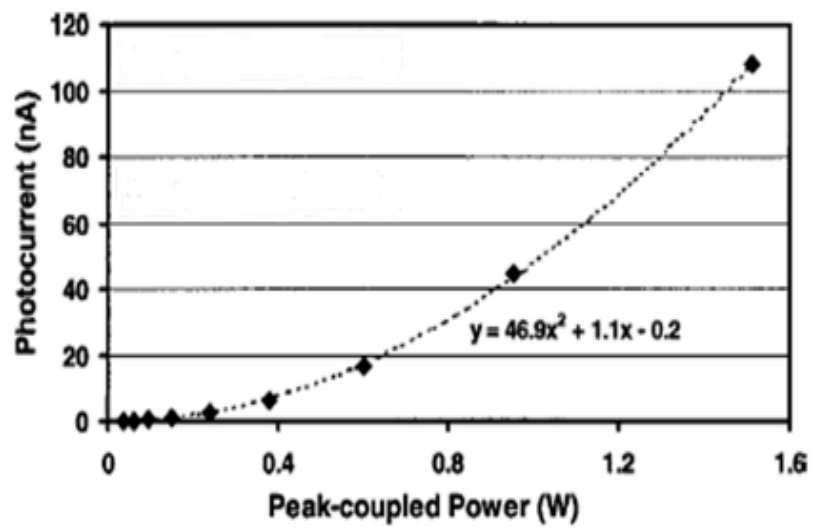

Figure 21. Sketch (a) and measured photocurrent (b) of the TPA-based detector proposed by Liang et al.[86]. Reprinted with permission from T. K. Liang et al., "Silicon waveguide two-photon absorption detector at $1.5 \mu \mathrm{m}$ wavelength for autocorrelation measurements," Appl. Phys. Lett., 81, 1323-1325. Copyright 2002, American Institute of Physics

In Figure 21b, the measured photocurrent for a bias voltage of $-3 \mathrm{~V}$ is reported and the typical non-linear behaviour of the TPA effect can be noted. Liang's device was used to perform an optical autocorrelation for measuring ultra-short laser pulses, but it was not suitable as a photodetector due to the high required optical input power value. The key to obtaining micrometer devices sensitive to low optical input is to strengthen the interaction between light and matter by tightly confining the light, i.e., employing high-Q microresonators. Bravo-Abad et al.[87] theoretically studied the feasibility of using $\mathrm{p}-\mathrm{i}-\mathrm{n}$ diode embedded silicon micro-ring or photonic crystal resonators for ultrafast photodetection. Starting from these premises, Tanabe et al.[88] proposed an infrared photodetector based on a $\mathrm{p}-\mathrm{i}-\mathrm{n}$ integrated into a high-Q width-modulated line-defect photonic crystal (PhC) nanocavity $[89,90]$. The sketch of the device proposed by the Japanese authors is reported in Fig. 22. The nanocavity is characterized by a lattice constant $\mathrm{a}=420 \mathrm{~nm}$, hole radius $\mathrm{r}=$ $108 \mathrm{~nm}$, slab thickness $\mathrm{t}=204 \mathrm{~nm}, \mathrm{~W}_{\mathrm{i}}=8.72 \mu \mathrm{m}$ and $\mathrm{W}_{\mathrm{w}}=$ $8.4 \mu \mathrm{m}$; whereas the input and output waveguides are $1.05 \mathrm{a}$ wide and are in-line connected with the cavity through barrier line defects $0.98 \mathrm{a}$ wide and $11 \mathrm{a}$ long.

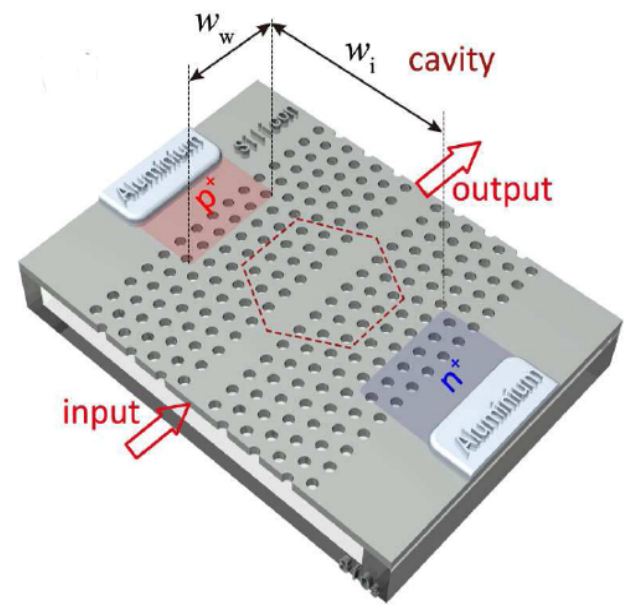

Figure 22. Schematic view of the device proposed by Tanabe et al.[88]. Reprinted with permission from T. Tanabe et al., "All-silicon sub-Gb/s telecom detector with low dark current and high quantum efficiency on chip," Appl. Lett., 96, 101103. Copyright 2010, American Institute of Physics

The $\mathrm{PhC}$ nanocavity is characterized by an intrinsic $\mathrm{Q}$ factor of $8.4 \times 105$. This high $\mathrm{Q}$ value permits the obtention of a high generation of photocurrent into the cavity region, enabling the possibility of detecting even low input power. In particular, an external quantum responsivity of $16 \mathrm{~mA} / \mathrm{W}$ at a resonance wavelength of aproximately $1500 \mathrm{~nm}$, has been measured. Furthermore, on the one hand, the very small dimensions of the device allow achievement of a low dark current value (about $15 \mathrm{pA}$ at room temperature for $3 \mathrm{~V}$ of reverse bias), and on the other hand, a small capacitance (a value of $9.5 \times 10^{-18} \mathrm{~F}$ was estimated). The resistance for the $\mathrm{p}$ and $\mathrm{n}$ regions limits the speed of the proposed device at 0.1 $\mathrm{GHz}$.

A silicon microdisk resonator with a laterally embedded p-i-n diode was chosen by Chen et al.[91] to enhance the TPA photocurrent. TPA is a very weak effect which is difficult to exploit for the realization of bulk all-silicon photodetectors with appreciable efficiency. For this reason, as shown in the literature, TPA exploitation in bulk silicon devices at $1550 \mathrm{~nm}$ is aimed at improving the sensitivity of two-photon absorption autocorrelators, utilizing, as the two-photon absorber, a silicon avalanche photodiode (APD) [92-94]. The avalanche process in APDs amplifies the weak IR two-photon absorption photocurrent, thereby greatly improving the sensitivity of the autocorrelation measurement. More recently, TPA in all-silicon bulk detectors has been exploited for a novel profilometry technique at $1.55 \mu \mathrm{m}[95]$; compared with conventional profilometry, TPA has a much wider dynamic range without the need for high-speed devices or complicated computation. Shi et al.[96] fabricated a hemispherical nearly-intrinsic silicon TPA photodetector operating at a wavelength of $1.3 \mu \mathrm{m}$ and emitted from a CW laser. The detector was made of nearly-intrinsic silicon crystal with a resistivity of $6000 \Omega / \mathrm{cm}$ and made into a hemisphere with a radius of $3 \mathrm{~mm}$. The silicon hemisphere was used as both a detector and a solid immersion lens (SIL) in the experiments. The bottom of the detector is (110) plane, on which aluminum electrodes were evaporated. The 
electrodes consisted of concentric circular and annular metal contacts with a spacing of $0.15 \mathrm{~mm}$ and a radius of $0.5 \mathrm{~mm}$ for the central electrode. The contacts between the aluminum and the silicon hemisphere are considered to be ohmic contacts. Because TPA substantially occurs in the vicinity of the focused spot (the center of the hemisphere), the concentric electrodes collect the photo-excited carriers efficiently.

The responsivity of the detector was about $2 \mu \mathrm{A} / \mathrm{W}$ at $1300 \mathrm{~nm}$ and $1 \mathrm{~V}$ of applied bias. This silicon photodetector is easy to fabricate and is useful in autocorrelation for measuring ultra-short laser pulses with wavelengths in the region of $1.2-2.1 \mu \mathrm{m}$.

\section{Conclusions}

In this work an overview on the NIR all-Si photodetectors has been presented. First, we have attempted to elucidate the most utilized physical effects allowing Si absorption at sub band-gap wavelengths such as: mid-bandgap absorption, surface state absorption, internal photoemission absorption and two photon absorption. Then a quantitative comparison of the photodetectors proposed in the scientific literature based on the aforementioned absorption mechanisms, including both bulk and integrated devices, have been reviewed. Overall, MBA based waveguide photodetectors seem to show the best performances if compared to devices based on others absorption mechanisms, however, new structures based on IPA are becoming very promising. Historically, photodetectors based on IPA are characterized by a low quantum efficiency. This is a direct result of many factors: 1) the low absorption due to high reflectivity of the metal layer at NIR wavelengths, 2) the conservation of momentum during carrier emission over the potential barrier which lowers the carriers emission probability into semiconductor, 3) the excitation of carriers lying in states far below the Fermi energy, which get very low probability to overcome the Schottky barrier. While the first and second points can be partially improved by using an optical cavity and a thin metal film or, as an alternative, plasmonc structures, the third point remains the main limiting factor of IPA-based devices. In 1971 Sheperd, Vickers and Yang[97] recommended replacing the metal electrodes IPE-based photodiodes with degenerate semiconductors. They reasoned that reducing the Fermi energy of electrode by substitution of a degenerate semiconductor could improve emission efficiency as much as 20-fold. Even if NIR IPA-based devices efficiency is still only adapted for power monitoring application, in our opinion that new structures based on the aforementioned insights could play a key role in telecommunication field and could open new frontiers in the field of low-cost silicon photonic.

\section{ACKNOWLEDGEMENTS}

I sincerely thank Dr. Giuseppe Coppola, Dr. Mario Iodice and Dr. Luigi Sirleto for fruitful discussions and feedback on the contents of this work.

\section{REFERENCES}

[1] Kimerling, L. C., Dal Negro, L., Saini, S., Yi, Y., Ahn, D.; Akiyama, S., Cannon, D., Liu, J., Sandland, J. G., Sparacin, D., Michel, J., Wada, K., Watts, M. R., 2004, Silicon Photonics: Topics in Applied Physics, Springer, Berlin.

[2] Jalali, B., and Fathpour, S., 2006, Silicon Photonics, J. Lightwave Technol., 24(12), 4600-4615.

[3] Rowe, L. K., Elsey, M., Tarr, N. G., Knights, A. P., Post, E., 2007, CMOS-compatible optical rib waveguides defined by local oxidation of silicon, Electron. Lett., 43(6), 392-393.

[4] Vivien, L., Pascal, D., Lardenois, S., Marris-Morini, D., Cassan, E., Grillot, F., Laval, S., Fedeli, J. M., El Melhaoui, L., 2006, Light injection in SOI microwaveguides using high-efficiency grating couplers, J. Lightw. Technol., 24(10), 3810-3815.

[5] Xu, Q., Manipatruni, S., Schmidt, B., Shakya, J., Lipson, M., 2007, $12.5 \mathrm{Gbit} / \mathrm{s}$ carrier-injection-based silicon micro-ring silicon modulators, Opt. Express, 15(2), 430-436.

[6] Michael, C. P., Borselli, M., Johnson, T.J., Chrystal, C., Painter, O., 2007, An optical fiber-taper probe for wafer-scale microphotonic device characterization, Opt. Express, 15(8), $4745-4752$.

[7] Liu, A., Jones, R., Cohen, O., Hak, D., Paniccia M., 2006, Optical amplification and lasing by stimulated Raman scattering in silicon waveguides, J. Lightw. Technol., 24(3), $1440-1445$

[8] Liu, A., Liao, L., Rubin, D., Nguyen, H., Ciftcioglu, B., Chetrit, Y., Izhaky, N., Paniccia, M., 2007, High-speed optical modulation based on carrier depletion in a silicon waveguide, Opt. Express, 15(2), 660-668.

[9] Schares, L., Schow, C., Doany, F., Schuster, C., Kash, J., Kuchta, D., Pepeljugoski, P., Schaub, J., Trewhella, J., Baks, C., John, R., Shan, L., Hegde, S., Kwark, Y., Rogers, D., Libsch, F., Budd, R., Chiniwalla, P., Rosner, J., Tsang, C., Patel, C., Kucharski, D., Guckenberge, D., Dangel, R., Offrein, B., Tan, M., Trott, G., Nystrom, M., Tandon, A., Lin, C.K., Dolfi, D., 2005, "Terabus" - A waveguide based parallel optical interconnect for $\mathrm{Tb} / \mathrm{s}$-class on-board data transfer in computer system. Proc. of 31 st European Conference on Optical Communications, 3, 369-372.

[10] Kartalopoulos, S.V., 2002, Elastic bandwidth, IEEE Circuits Devices, 18, 8-13.

[11] Schaub, J.D., Li, R., Csutak, S.M., Campbell, J.C., 2001, High-speed monolithic silicon photoreceivers on high resistivity and SOI substrates, J. Lightw. Tech., 19, 272-278.

[12] Fan, H. Y., and Ramdas, A. K., 1959, Infrared Absorption and Photoconductivity in Irradiated Silicon, Journal of Applied Physics, 30, 1127-1134.

[13] Wertheim, G. K., 1957, Energy Levels in Electron-Bombarded Silicon, Phys. Rev., 105, 1730. 
[14] Hill, D. E., 1958, Ph.D. thesis, Purdue University.

[15] Longo, T. A., 1957, Ph.D. thesis, Purdue University.

[16] Fan, H. Y., and Lark-Horovitz, K., 1958, Effects of radiation on Materials, Harwood, Hausner, Morse and Rauch: New York.

[17] Lark-Horovitz, K., 1951, Semi-Conducting Materials, H. K. Henisch: London.

[18] Wertheim G. K., 1958, Neutron-Bombardment Damage in Silicon, Phys. Rev., 111, 1500-1505.

[19] Loferski, J. J., and Rappaport, P., 1958, Radiation Damage in Ge and Si Detected by Carrier Lifetime Changes: Damage Thresholds, Phys. Rev., 111, 432-439.

[20] Geis, M. W., Spector, S. J., Grein, M. E., Schulein, R.T., Yoon, J. U., Lennon, D. M., Wynn, C. M., Palmacci, S. T., Gan, F., Kärtner, F. X., Lyszczarz, T. M., 2007, All silicon infrared photodiodes: Photo response and effects of processing temperature, Opt. Express, 15, 16886-16895.

[21] Libertino, S., Coffa, S., Benton, J. L., Halliburton, K., Eaglesham, D. J., 2001, Formation, evolution and annihilstion of interstitial clusters in ion implanted Si, Phys. Rev. B, 63, 195206.

[22] Harrick, N. J., 1962, Optical Spectrum of the Semiconductor Surface States from Frustrated Total Internal Reflections, Phys. Rev., 125, 1165.

[23] Samoggia G., Nuciotti A., Chiarotti G., 1996, Optical Detection of Surface States in Ge, Phys. Rev., 144, 749.

[24] Chiarotti, G., Del Signore, G., Nannarone, S., 1968, Optical Detection of Surface States on Cleaved (111) Surfaces of Ge., Phys. Rev. Letters, 21, 1170.

[25] Allen, F. C., and Gobeli, G. W., 1962, Work Function, Photoelectric Threshold, and Surface States of Atomically Clean Silicon, Phys. Rev., 127, 150.

[26] Garcia-Moliner, F., and Rubio, J., 1969, A new method in the quantum theory of surface states, J. Phys. C: Solid State Phys., 2,1789 .

[27] Bortolani, V., Calandra, C., Sghedoni, A., 1971, Surface states in Si, Phys. Letters A, 34, 193.

[28] Schocley, W., 1939, On the Surface States Associated with a Periodic Potential., Phys. Rev., 56, 317-323.

[29] Chiarotti, G., Nannarone, S., Pastore, R., Chiaradia, P., 1971, Optical Absorption of Surface States in Ultrahigh Vaccum Cleaved (111) Surfaces of Ge and Si, Physical Review B, 4, 3398-3402.

[30] Fowler, R. H., 1931, The analysis of photoelectric sensitivity curves for clean metals at various temperatures, Phys. Rev., $38,45-56$

[31] Vickers, V. E., 1971, Model of schottky barrier hot-electron-mode photodetection, Appl. Opt., 10, 2190-2192.

[32] Chan, E. Y., Card, H. C., Teich, M. C., 1980, Internal Photoemission Mechanism at interfaces between Germanium and Thin Metal Films, IEEE J. Quantum Electron., 16, 373-381.

[33] Scales, C., and Berini, P., 2010, Thin-film Schottky barrier
Photodetector Models, IEEE Journal of Quantum Electronics, 46(5), 633-643.

[34] Sze, S. M., 1981, Physics of Semiconductor Devices; John Wiley \& Sons, New York.

[35] Yuan H. X., and Perera, A. G. U., 1995, Dark current analysis of Si homojunction interfacial work function internal photoemission far-infrared detectors, Appl. Phys. Lett., 66, $2262-2264$

[36] Boggess, T. F., Bohnert, K. M., Mansour, K., Moss, S. C., Boyd, I. W., Smirl, A. L., 1986, Simultaneous measurement of two-photon coefficient and free-carrier cross section above the bandgap of crystalline silicon, IEEE J. Quantum Electron., $22,360-368$.

[37] Reintjes J. F., McGroddy, J. C., 1973, Indirect two-photon transitions in Si at $1.06 \mu \mathrm{m}$, Phys. Rev. Lett., 30, 901-903.

[38] Reitze, D. H., Zhang, T. R., Wood, W. M., Downer, M. C., 1990, Two-photon spectroscopy of silicon using femtosecond pulses at above-gap frequencies, J. Opt. Soc. Am. B, 7, 84-89.

[39] Tsang, H. K., Wong, C. S., Liang, T. K., Day, I. E., Roberts, S. W., Harpin, A., Drake, J., Asghari, M., 2002, Optical dispersion, two-photon absorption and self-phase modulation in silicon waveguides at $1.5 \mu \mathrm{m}$ wavelength, Appl. Phys. Lett., 80(3), 416-418.

[40] Cowan, A. R., Rieger, G. W., Young, J. F., 2004, Nonlinear transmission of $1.5 \mu \mathrm{m}$ pulses through single-mode silicon-on-insulator waveguide structures, Opt. Express, 12, $1611-1621$

[41] Dinu, M., Quochi, F., Garcia, H., 2003, Third-order nonlinearities in silicon at telecom wavelengths, Appl. Phys. Lett., 82, 2954-2956.

[42] Dinu, M., 2003, Dispersion of phonon-assisted nonresonant third-order nonlinearities, IEEE J. Quantum Electron., 39, 1498-1503.

[43] Bristow, A. D., Rotenberg, N., van Driel H. M., 2007, Two-photon absorption and Kerr coefficients of silicon for 850-2200 nm., Appl. Phys. Lett., 90, 191104.

[44] Euser, T. G., Vos, W. L., 2005, Spatial homogeneity of optically switched semiconductor photonic crystals and of bulk semiconductors, J. Appl. Phys., 97, 043102.

[45] Garcia H., and Kalyanaraman, R., 2006, Phonon-assisted two-photon absorption in the presence of a dc-field: the nonlinear Franz-Keldysh effect in indirect gap semiconductors, J. Phys. B, 39, 2737-2746.

[46] Knights, A., House, A., MacNaughton, R., Hopper, F., 2003, Optical power monitoring function compatible with single chip integration on silicon-on-insulator Proc. of conference on Optical Fiber Communication, Technical Digest Series, 2, 705-706.

[47] Bradley, J. D. B., Jessop, P. E., Knights, A. P., 2005, Silicon waveguide-integrated optical power monitor with enhanced sensitivity at $1550 \mathrm{~nm}$, Appl. Phys. Lett., 86, 241103.

[48] Knights, A. P., Bradley, J. D., Gou, S. H., Jessop, P. E., 2006, Silicon-on-insulator waveguide photodetector with self-ion-implantation-engineered enhanced infrared response, J. Vac. Sci. Technol. A, 24, 783-786. 
[49] Liu, Y., Chow, C. W., Cheung, W. Y., Tsang, H. K., 2006, In-line channel power monitor based on helium ion implantation in silicon-on-insulator waveguides, IEEE Phot. Technol. Lett., 18, 1882-1884.

[50] Giri, P. K., and Mohapatra, Y. N., 2000, Thermal stability of defect complexes due to high dose MeV implantation in silicon, Mater. Sci. Eng., 71, 327-332.

[51] Geis, M. W., Spector, S. J., Grein, M. E., Schulein, R. T., Yoon, J. U., Lennon, D. M., Denault, S., Gan, F., Kaertner, F. X., Lyszczarz, T. M., 2007, CMOS-compatible all-Si high-speed waveguide photodiodes with high responsivity in near-infrared communication band, IEEE Photon. Technol. Lett., 19, 152-154.

[52] Geis, M. W., Spector, S. J., Grein, M. E., Yoon, J. U., Lennon, D. M., Lyszczarz, T. M., 2009, Silicon waveguide infrared photodiodes with $>35 \mathrm{GHz}$ bandwidth and phototransistors with 50 AW-1 response, Opt. Express, 17, 5193-5204.

[53] Shafiiha, R., Zheng, D., Liao, S., Dong, P., Liang, H., Feng, N., Luff, B. J., Feng, D., Li, G., Cunningham, J., Raj, K., Krishnamoorthy, A. V., Asghari, M., 2010, Silicon waveguide coupled resonator infrared detector. Proc. of Optical Fiber Communication Conference, 21-25.

[54] Almeida, V. R., Barrios, C. A., Panepucci, R. R., Lipson, M., Foster, M. A., Ouzonnov, D. G., Gaeta, A. L., 2004, L-optical switching on a silicon chip, Opt. Lett., 29, 2867-2869.

[55] Baehr-Jones, T., Hochberg, M., Walker, C., Scherer, A., 2004, High-Q ring resonators in thin silicon-on-insulator, Appl. Phys. Lett., 85, 3346-3347.

[56] Little, B. E., Chu, S. T., Haus, H. A., Foresi, J., Laine, J. P., 1997, Microring resonator channel dropping filters, J. Lightw. Technol., 15, 998-1005.

[57] Doylend J. K., Jessop P. E., Knights A. P., 2010, Silicon photonic resonator-enhanced defect-mediated photodiode for sub-bandgap detection, Opt. Express, 18, 14671-14678.

[58] Wu, C., Crouch, C. H., Zhao, L., Carey, J. E., Younkin, R., Levinson, J. A., Mazur E., Farrell, R. M., Gothoskar, P., Karger, A., 2001, Near-unity below-band-gap absorption by microstructured silicon, Appl. Phys. Lett., 78, 1850-1852.

[59] Carey, J. E., Crouch, C. H., Shen, M., Mazur. E., 2005, Visible and near-infrared responsivity of femtosecond-laser microstructured silicon photodiodes, Opt. Lett., 30, 1773-1775.

[60] Baehr-Jones, T., Hochberg, M., Scherer, A., 2008, Photodetection in silicon beyond the band edge with surface states, Opt. Express, 16, 1659-1668.

[61] Chen, H., Luo, X., Poon, A. W., 2009, Cavity-enhanced photocurrent generation by $1.55 \mu \mathrm{m}$ wavelengths linear absorption in a $\mathrm{p}-\mathrm{i}-\mathrm{n}$ diode embedded silicon microring resonator, Appl. Phys. Lett., 95, 171111.

[62] Kosonocky, W. F., Shallcross, F. V., Villani, T. S., 1985, 160x244 Element PtSi Schottky-Barrier IR-CCD Image Sensor, IEEE Trans. Electron Dev., ED-32( 8), 1564.

[63] Casalino, M., Sirleto, L., Moretti, L., Della Corte, F., Rendina, I., 2006, Design of a silicon RCE schottky photodetector working at 1.55 micron, Journal of luminescence, 121, 399-402.

[64] Casalino, M., Sirleto, L., Moretti, L., Della Corte, F., Rendina,
I., 2006, Design of a silicon resonant cavity enhanced photodetector based on the internal photoemission effect at $1.55 \mu \mathrm{m}$. Journal of Optics A: Pure and applied optics, 8, 909-913.

[65] Casalino, M., Sirleto, L., Moretti, L., Rendina, I., 2008, A silicon compatible resonant cavity enhanced photodetector working at $1.55 \mu \mathrm{m}$, Semicond. Sci. Technol., 23(7), 075001.

[66] Elabd, H., Villani, T., Kosonocky, W. F., 1982, Palladium-Silicide Schottky-Barrier IR-CCD for SWIR Applications at Intermediate Temperatures, IEEE Trans. Electron Devices Lett., EDL-3, 89-90.

[67] Lee, M. K., Chu, C. H., Wang Y. H., 2001, 1.55- $\mu \mathrm{m}$ and infrared-band photoresponsivity of a Schottky barrier porous silicon photodetector, Opt. Lett., 26(3), 160-162.

[68] Casalino, M., Sirleto, L., Moretti, L., Gioffrè, M., Coppola, G., Rendina, I., 2008, Silicon resonant cavity enhanced photodetector based on the internal photoemission effect at $1.55 \mu \mathrm{m}$ : Fabrication and characterization, Appl. Phys. Lett., 92(25), 251104.

[69] Casalino, M., Coppola, G., Gioffrè, M., Iodice, M., Moretti, L., Rendina, I., Sirleto, L., 2010, Cavity enhanced internal photoemission effect in silicon photodiode for sub-bandgap detection, J. Lightw. Technol., 28(22), 3266-3272

[70] Casalino, M., Sirleto, L., Iodice, M., Saffioti, N., Gioffrè, M., Rendina, I., Coppola, G., 2010, Cu/p-Si Schottky barrier-based near infrared photodetector integrated with a silicon-on-insulator waveguide, Appl. Phys. Lett., 96(24), 241112 .

[71] Zhu, S., Yu, M. B., Lo, G. Q., Kwong, D. L., 2008, Near-infrared waveguide-based nickel silicide Schottky-barrier photodetector for optical communications, Appl. Phys. Lett., 92(8), 081103.

[72] Zhu, S., Lo, G. Q., Kwong, L., 2008, Low-cost and high-gain silicide Schottky-barrier collector phototransistor integrated on Si waveguide for infrared detection, Appl. Phys. Lett., 93(7), 071108

[73] Zhu, S., Lo, G. Q., Kwong, D. L., 2008, Low-Cost and High-Speed SOI Waveguide-Based Silicide Schottky-Barrier MSM Photodetectors for Broadband Optical Communications, IEEE Phot. Tech. Lett., 20(16), 1396-1398

[74] Reather, H., 1988, Surface Plasmons on Smooth and Rough Surfaces and on Gratings. Springer, Berlin.

[75] Ebbesen, W., Lezec, H. J., Ghaemi, H. F., Thio, T., Wolff, P. A., 1997, Extraordinary optical transmission through sub-wavelength hole arrays, Nature, 391, 667-669.

[76] Barnes, W. L., Dereux, A., Ebbesen, T. W., 2003, Surface plasmon subwavelength optics. Nature, 424(6950), 824-830.

[77] Maier A., 2006, Plasmonics: Fundamentals and Applications. Springer, New York.

[78] Scales, C., and Berini, P., 2004, Schottky Barrier Photodetectors, U. S. Patent No. 7,026,701.

[79] Scales, C., Breukelaar, I., Berini, P., 2009, Surface-plasmon Schottky contact detector based on a symmetric metal stripe in silicon, Opt. Lett., 35(4), 529-531.

[80] Scales, C., Breukelaar, I., Charbonneau, R., Berini, P., 2011, 
Infrared Performance of Symmetric Surface-Plasmon Waveguide Schottky Detectors in Si, IEEE J. Lightw. Tech., 29(12), 1852-1860.

[81] Akbari A., and Berini, P., 2009, Schottky contact surface-plasmon detector integrated with an asymmetric metal stripe waveguide, Appl. Phys. Lett., 95(2), 021104.

[82] Akbari, A., Tait, R. N., Berini, P., 2010, Surface plasmon waveguide Schottky detector, Opt. Express, 18(8), 85058514.

[83] Olivieri, A., Akbari A., and Berini, P., 2010. Surface plasmon waveguide Schottky detectors operating near breakdown, Phys. Status Solidi RRL, 4(10), 283 - 285.

[84] Goykhman, I., Desiatov, B., Khurgin, J., Shappir, J., Levy U., 2011, Locally Oxidized Silicon Surface-Plasmon Schottky Detector for Telecom Regime, Nano Lett., 11, 2219-2224.

[85] Tsang, H. K., and Liu, Y., 2008, Nonlinear optical properties of silicon waveguides, Semicond. Sci. Technol., 23, 064007.

[86] Liang, T. K., Tsang, H. K., Day, I. E., Drake, J., Knights, A. P., Asghari, M., 2002, Silicon waveguide two-photon absorption detector at $1.5 \mu \mathrm{m}$ wavelength for autocorrelation measurements, Appl. Phys. Lett., 81, 1323-1325.

[87] Bravo-Abad, J.; Ippen, E.P.; Soljačić, M. Ultrafast photodetection in an all-silicon chip enabled by two-photon absorption. Appl. Phys. Lett. 2009, 94, 241103.

[88] Tanabe, T., Sumikura, H., Taniyama, H., Shinya, A., Notomi, M., 2010, All-silicon sub-Gb/s telecom detector with low dark current and high quantum efficiency on chip, Appl. Phys. Lett., 96, 101103.
[89] Akahane, Y., Asano, T., Song, B. S., Noda, S., 2003, High-Q photonic nanocavity in a two-dimensional photonic crystal, Nature, 425, 944-947.

[90] Tanabe, T., Nishiguchi, K., Kuramochi, E., Notomi, M., 2009, Low power and fast electro-optic silicon modulator with lateral p-i-n embedded photonic crystal nanocavity, Opt. Express, 17, 22505-22513.

[91] Chen H., and Poon, A. W., 2010, Two-photon absorption photocurrent in $\mathrm{p}$-i-n diode embedded silicon microdisk resonators. Appl. Phys. Lett., 96, 191106.

[92] Kikuchi, K., 1998, Highly sensitive interferometric autocorrelator using Si avalanche photodiode as two-photon absorber. IEEE Elec. Lett., 34, 123-125.

[93] Kikuchi, K., 1998, Optical sampling system at $1.5 \mu \mathrm{m}$ using two photon absorption in $\mathrm{Si}$ avalanche photodiode. IEEE Elec. Lett., 34, 1354-1355.

[94] Salem, R., and Murphy, T. E., 2004, Polarization-insensitive cross correlation using two-photon absorption in a silicon photodiode, Opt. Lett., 29, 1524-1526.

[95] Tanaka, Y., Sako, N., Kurokawa, T., 2003, Profilometry based on two-photon absorption in a silicon avalanche photodiode, Opt. Lett., 28, 402-404.

[96] Shi, B., Liu, X., Chen, Z., Jia, G., Cao, K., Zhang, Y., Wang, S., Ren, C., Zhao, J., 2008, Anisotropy of photocurrent for two-photon absorption photodetector made of hemispherical silicon with (110) plane, Appl. Phys. B, 93, 873-877.

[97] Sheperd, F. D., Vickers, V. E., Yang, A. C., 1971, Schottky Barrier Photodiode with a Degenerate Semiconductor Active Region, U.S. Patent No. 3.603.847. 